\title{
Interferon-driven brain phenotype in a mouse model of RNaseT2 deficient leukoencephalopathy
}

\author{
Matthias Kettwig (1) 1,18凶 Katharina Ternka (10) ${ }^{1,18}$, Kristin Wendland ${ }^{1}$, Dennis Manfred Krüger²,

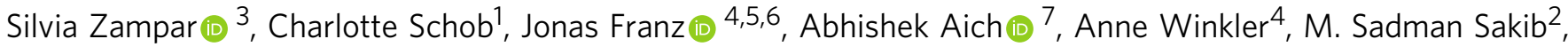 \\ Lalit Kaurani $^{2}$, Robert Epple ${ }^{2}$, Hauke B. Werner (10 ${ }^{8}$, Samy Hakroush ${ }^{9}$, Julia Kitz ${ }^{9}$, Marco Prinz (D) 10,11,12, \\ Eva Bartok (10) 14, Gunther Hartmann ${ }^{13}$, Simone Schröder ${ }^{1}$, Peter Rehling ${ }^{7}$, Marco Henneke , \\ Susann Boretius (D) ${ }^{15}$, A. Alia ${ }^{16,17}$, Oliver Wirths (1D ${ }^{3}$, Andre Fischer ${ }^{2,3}$, Christine Stadelmann (D) ${ }^{4}$, \\ Stefan Nessler ${ }^{4,19}$ \& Jutta Gärtner ${ }^{1,19}$
}

Infantile-onset RNaseT2 deficient leukoencephalopathy is characterised by cystic brain lesions, multifocal white matter alterations, cerebral atrophy, and severe psychomotor impairment. The phenotype is similar to congenital cytomegalovirus brain infection and overlaps with type I interferonopathies, suggesting a role for innate immunity in its pathophysiology. To date, pathophysiological studies have been hindered by the lack of mouse models recapitulating the neuroinflammatory encephalopathy found in patients. In this study, we generated Rnaset2 ${ }^{-/-}$mice using CRISPR/Cas9-mediated genome editing. Rnaset2-1mice demonstrate upregulation of interferon-stimulated genes and concurrent IFNAR1dependent neuroinflammation, with infiltration of $\mathrm{CD}^{+}$effector memory $\mathrm{T}$ cells and inflammatory monocytes into the grey and white matter. Single nuclei RNA sequencing reveals homeostatic dysfunctions in glial cells and neurons and provide important insights into the mechanisms of hippocampal-accentuated brain atrophy and cognitive impairment. The Rnaset2 ${ }^{-/-}$mice may allow the study of CNS damage associated with RNaseT2 deficiency and may be used for the investigation of potential therapies.

\footnotetext{
${ }^{1}$ Department of Pediatrics and Adolescent Medicine, Division of Pediatric Neurology, University Medical Center Göttingen, Georg August University, Göttingen, Germany. ${ }^{2}$ Department for Epigenetics and Systems Medicine in Neurodegenerative Diseases, German Center for Neurodegenerative Diseases (DZNE), Göttingen, Germany. ${ }^{3}$ Department of Psychiatry and Psychotherapy, University Medical Center Göttingen, Georg August University,

Göttingen, Germany. ${ }^{4}$ Institute of Neuropathology, University Medical Center Göttingen, Georg August University, Göttingen, Germany. ${ }^{5}$ Campus Institute for Dynamics of Biological Networks, University of Göttingen, Göttingen, Germany. ${ }^{6}$ Max Planck Institute for Experimental Medicine, Göttingen, Germany. ${ }^{7}$ Department of Cellular Biochemistry, University Medical Center Göttingen, Georg August University, Göttingen, Germany. ${ }^{8}$ Department of Neurogenetics, Max Planck Institute of Experimental Medicine, Göttingen, Germany. ${ }^{9}$ Institute of Pathology, University Medical Center Göttingen, Georg August University, Göttingen, Germany. ${ }^{10}$ Institute of Neuropathology, Faculty of Medicine, University of Freiburg, Freiburg, Germany. ${ }^{11}$ Signalling Research Centres BIOSS and CIBSS, University of Freiburg, Freiburg, Germany. ${ }^{12}$ Center for Basics in NeuroModulation (NeuroModulBasics), Faculty of Medicine, University of Freiburg, Freiburg, Germany. ${ }^{13}$ Institute of Clinical Chemistry and Clinical Pharmacology, University Hospital, University of Bonn, Bonn, Germany. ${ }^{14}$ Unit of Experimental Immunology, Department of Biomedical Sciences, Institute of Tropical Medicine, Antwerp, Belgium. ${ }^{15}$ Functional Imaging Laboratory, German Primate Center, Leibniz Institute for Primate Research, Göttingen, Germany. ${ }^{16}$ Institute for Medical Physics and Biophysics, University of Leipzig, Leipzig, Germany. ${ }^{17}$ Leiden Institute of Chemistry, Leiden University, Leiden, The Netherlands. ${ }^{18}$ These authors contributed equally: Matthias Kettwig, Katharina Ternka. ${ }^{19}$ These authors jointly supervised this work: Stefan Nessler, Jutta Gärtner. ${ }^{凶}$ email: matthias.kettwig@med.uni-goettingen.de
} 
uman RNaseT2 deficiency is a rare, monogenetic, early childhood onset cystic leukoencephalopathy (CLE) which manifests as psychomotor delay, spasticity, epilepsy, and normo- or microcephaly during the first year of life. Brain magnetic resonance imaging (MRI) reveals frontal and temporal lobe cystic lesions, multifocal white matter alterations, and cerebral atrophy ${ }^{1,2}$. The phenotypic features of RNaseT2-deficient CLE are indistinguishable from the sequelae of in utero cytomegalovirus $(\mathrm{CMV})$ brain infection. Moreover, there is a significant clinical and neuroradiological overlap of RNaseT2-deficient CLE with Aicardi-Goutières syndrome (AGS), with some affected individuals demonstrating cerebrospinal fluid (CSF) pleocytosis, elevated levels of CSF neopterin as an inflammatory marker, and an overexpression of interferon-stimulated genes (ISGs) in peripheral blood ${ }^{3}$.

Congenital viral brain infections are characterized by increased concentrations of type I interferons (IFN-I) in serum and CSF ${ }^{4,5}$. IFN-I is an essential antiviral cytokine, which induces a broad range of antiviral effector genes and inhibits cellular transcription, translation, and proliferation as well as enhancing cross presentation and driving clonal $\mathrm{T}$ cell expansion ${ }^{6,7}$. However, despite its vital function, chronic or constitutive IFN-I release can also cause devastating autoinflammation, particularly in the central nervous system $(\mathrm{CNS})^{4}$. While mice with transgenic constitutive IFN-I expression from astrocytes are exceptionally resistant to neurotropic viruses, they demonstrate progressive inflammatory encephalopathy, including cerebral calcification ${ }^{8,9}$. Similarly, IFN-I in the CSF is a hallmark of AGS and considered the principle driver of the severe autoinflammatory encephalopathy observed in these patients.

IFN release is triggered by the activation of innate anti-viral pattern recognition (immune sensing) receptors (PRR) during viral infection, many of which sense viral nucleic acids ${ }^{10}$. However, other triggers such as the mislocalization or modification of self nucleic acids can also activate these pathways ${ }^{10,11}$. In particular, defects in genes involved in nucleic acid immune sensing, nucleic acid editing, and nucleic acid degradation can lead to inappropriate, chronic IFN-I release, known as type I interferonopathy ${ }^{12}$. AGS is the prototype of this disease, with the clinical hallmarks of severe psychomotor retardation, brain white matter abnormalities, and cerebral calcifications. Affected individuals can also present with extraneurological features, such as hepatosplenomegaly, inflammatory bowel disease, systemic lupus erythematosus and abnormalities of hematopoiesis ${ }^{13-15}$.

A number of the genes associated with type I interferonopathy and AGS code for nucleases, including TREX1, RNASEH2A, RNASEH2B, RNASEH2C, DNASE2, and SKIV2L. Hypomorphic variants of these genes lead to defects in nuclease activity and thus the accumulation of self nucleic acids and erroneous activation of anti-viral nucleic acid immune sensing receptors ${ }^{12,16}$. In line with these observations, RNaseT2 deficient CLE is caused by lossof-function mutations of the RNASET2 gene, a member of the endoribonuclease family that consists of RNase A, RNase T1, and RNase T2. RNaseT2 is exceptionally well conserved across species, including bacteria, yeast and plants and is involved in the endosomal degradation of RNA from mitochondria and efferocytosed cells ${ }^{17-20}$. It has been shown that RNaseT2 activity induces type I interferon signaling by degrading longer exogenous RNA molecules into ligands for the pattern recognition receptor TLR8 $8^{21,22}$.

In the last decade, mouse models for inborn type I interferonopathies, including all currently known AGS genes, have been generated to gain insights into disease mechanisms (Supplementary Table 1). Notably, other than the microglia-specific Usp $18^{-1-}$ mouse, none of these models were reported to display neuroinflammation or other neuropathologies. In Usp $18^{-/-}$mice, the missing negative regulator of Stat1 signaling leads to a tonically active IFN-I signal that causes a destructive white matter microgliopathy ${ }^{23}$. However, some models (Trex1, Adar1, and Ifih1 mutant mice) still demonstrated inflammatory and autoimmune phenotypes outside the CNS, such as myocarditis, inflammatory liver disease, lupus-like nephritis, and other systemic autoimmune symptoms in combination with impaired murine hematopoiesis ${ }^{24-27}$. In contrast, Samhd1-deficient mice are healthy, despite displaying an interferon signature in distinct tissues and cells, while Rnaseh2-deficient mice generally die on embryonic day 9.5 without inflammatory changes ${ }^{28,29}$.

In this work, we present the first murine model of inborn RNaseT2 deficiency. Strikingly, in contrast to almost all other mouse models of type I interferonopathy, Rnaset $2^{-1-}$ mice demonstrate type I IFN upregulation in the CNS and distinct CNS abnormalities that resemble characteristics of the human disease including brain atrophy and cognitive impairment. Outside the CNS, Rnaset $2^{-l-}$ mice recapitulate prominent features of known AGS models such as lymphocytic infiltration and the presence of an interferon signature in various organs, disturbed hematopoiesis, and autoantibody formation. As the first described whole-body mouse model of type I interferonopathy with a CNS phenotype, the Rnaset $2^{-1-}$ mouse provides important insights into the mechanisms of IFN-Idependent neurodegeneration. Moreover, Rnaset $2^{-1-}$ mice might contribute to develop novel therapeutic approaches for IFN-mediated CNS inflammation.

\section{Results}

Endonuclease-mediated generation of Rnaset $2 \mathrm{a}^{\mathrm{tm} 1(\mathrm{KOMP}) \mathrm{Wtsi}}$ Rnaset $2 \mathrm{~b}^{\mathrm{em} 1 \mathrm{Gaer}}$ double knockout mice (Rnaset2-/-). Two orthologues of the single human RNASET2 gene-Rnaset $2 a$ and Rnaset $2 b$, are present in close proximity on chromosome 17 in the mouse genome. Interestingly, they show minor base pair differences at the DNA level, whereas at the protein level, the two Rnaset2 orthologues are identical. Due to their proximity, generating double-knockout mice by classical interbreeding of Rnaset $2 a$ and Rnaset $2 b$ single-knockout mice was unfeasible ${ }^{30}$. However, using CRISPR/Cas9 mediated genome editing, we were able to generate the RNT2AB-double mutant mice by modifying the Rnaset $2 b$ locus in the background of the Rnaset2atm1(KOMP)Wtsi (RNT2A-KO) (Supplementary Fig. 1a). In brief, the Rnaset $2^{-1-}$ was generated by injecting CRISPR/Cas9 reagents into oocytes of RNT2A-KO mice. Founder lines were screened for an early stop codon at the end of exon 2 in the Rnaset $2 b$ locus and for the transgenic cassette (EUCOMM) in the Rnaset $2 a$ gene by PCR and Sanger sequencing (Supplementary Fig. 1a). Line 7 was backcrossed with C57BL/6N wildtype (WT) mice to generate N1 F0 heterozygous mice and then further expanded. Deficiency of RNaseT2a and b on protein level in

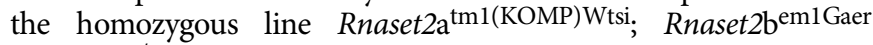
(Rnaset $^{-I-}$ ) was confirmed by immunoblot analysis of embryonic fibroblasts (MEFs) (Supplementary Fig. 1b). WT, heterozygous (Rnaset $2^{+/-}$) and homozygous (Rnaset ${ }^{-/-}$) mice were born in the expected Mendelian ratio and homozygous Rnaset $2^{-1-}$ mice were viable and fertile.

Rnaset $2^{-I-}$ mice have a reduced life expectancy. At the age of weaning at 3 weeks, Rnaset $2^{-1-}$ mice were indistinguishable from heterozygous or wild-type siblings regarding their feeding and social behavior, nesting instinct and activity (not quantified). At $\sim 10$ weeks of age, Rnaset $2^{-1-}$ mice displayed an enlarged abdominal girth due to hepatosplenomegaly. At this age, most Rnaset $2^{-1-}$ mice showed slightly reduced activity but no evidence of pain. For animal welfare reasons the Rnaset $2^{-/-}$mice were frequently scored by animal caretakers and veterinarians with 

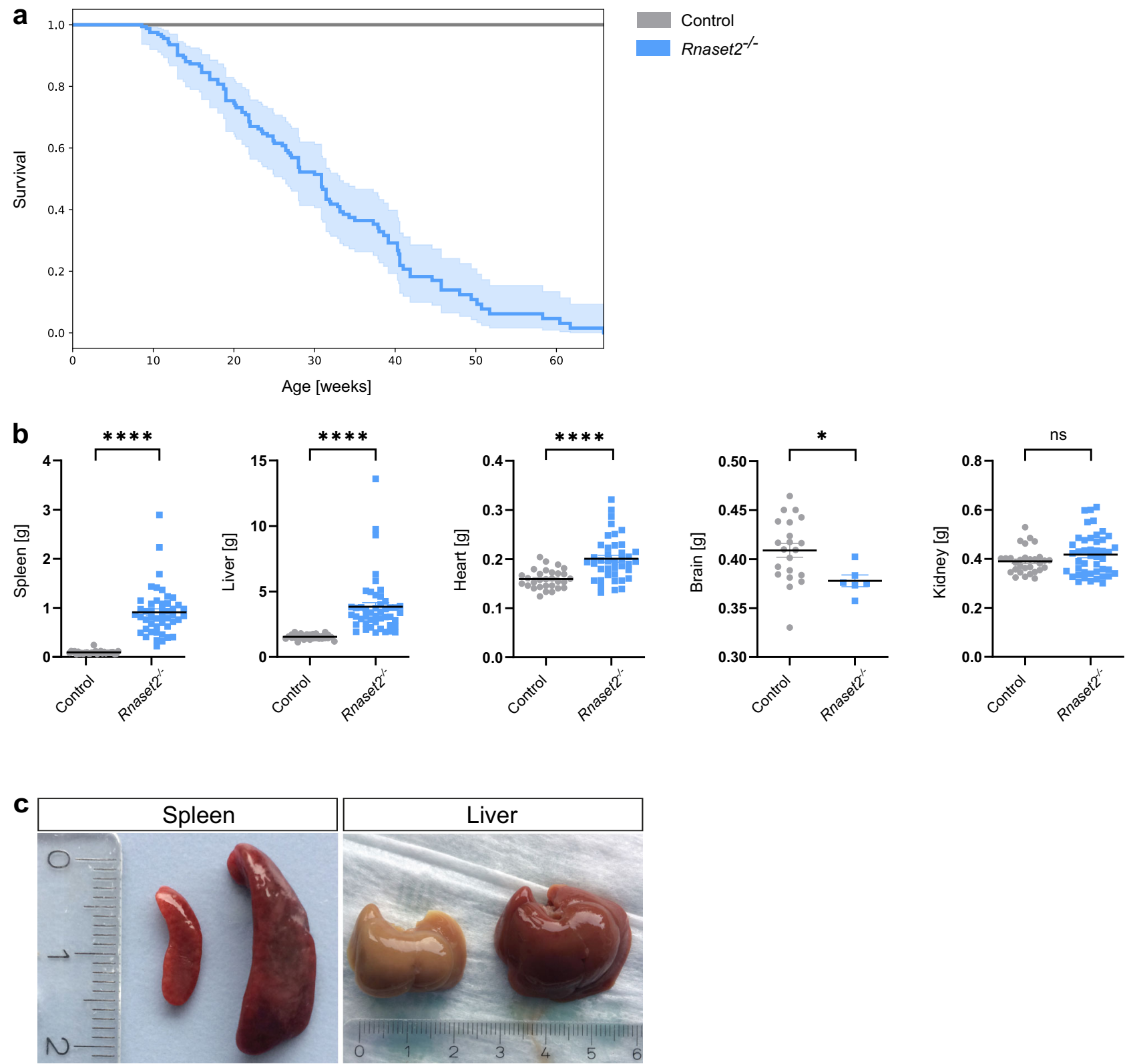

Fig. 1 Survival and organ enlargement of Rnaset2 ${ }^{-/-}$mice. a Kaplan-Meier blot survival for Rnaset2 ${ }^{-/-}$(blue, $n=173$ ) and control (gray, $n=102$ ) mice. The median lifetime of Rnaset2-/- mice is 31 weeks. For the survival curve, a confidence interval of $95 \%$ is estimated using the exponential Greenwood method. The alpha level was corrected using Bonferroni's method by the number of comparisons (alpha $=0.05 / 3$ ). b Comparison of organ weights from Rnaset ${ }^{-/}$mice ( $n=47$ for spleen and liver, $n=40$ for heart, $n=45$ for kidney and $n=6$ for brain) and from control mice ( $n=30$ for spleen, liver, heart and kidney and $n=21$ for brain). Rnaset2 $2^{-/-}$mouse revealed a significant enlargement of spleen, liver, and heart ( $p<0.0001$, respectively). Brain weight was significantly reduced $(p=0.0336)$ and kidney remained unaltered $(p=0.1205)$. Data are represented as mean values \pm SEM and $p$ values are depicted as ${ }^{\star \star \star \star} p<0.0001,{ }^{\star} p<0.05$ and not significant $p \geq 0.05$. c Macroscopy picture of Rnaset ${ }^{-1}$ - and control spleen (left) and liver (right) after PBS perfusion in 5-month-old representative mice. Rnaset $2^{-/}$spleen and liver were enlarged in volume and showed a dark red color. Source data are provided as a Source Data file.

a score sheet adapted to this particular animal model. The reporting of animals reaching the predefined endpoint criteria was genotype-blinded. The determined median lifetime of Rnaset $2^{-1-}$ mice was 31 weeks (Fig. 1a).

Upon autopsy, organ weight analysis of Rnaset $2^{-1-}$ mice revealed approximately 10 -fold enlarged spleens $(0.91 \pm 0.48$ vs. $0.09 \pm 0.03 \mathrm{~g}), 2.5$-fold enlarged livers $(3.84 \pm 2.19$ vs. $1.55 \pm 0.19 \mathrm{~g})$ and moderately enlarged hearts $(0.20 \pm 0.04$ vs. $0.16 \pm 0.02 \mathrm{~g})$, whereas their brains were significantly decreased in weight $(0.38 \pm 0.01$ vs. $0.41 \pm 0.03 \mathrm{~g})$. The kidney weight remained unchanged (Fig. 1b). Notably, liver and spleen had a dark red color despite extensive perfusion with phosphate-buffered saline (PBS) (Fig. 1c), indicative of extramedullary hematopoiesis.

Rnaset $2^{-/-}$mice develop autoinflammatory organ disease and disturbed hematopoiesis. In line with the dark red color observed (Fig. 1c), the spleen of Rnaset $2^{-1-}$ mice showed massive extramedullary hematopoiesis (Supplementary Fig. 2a-e). Furthermore, histological evaluations demonstrated an increased white splenic pulp (Fig. 2a) and predominantly lymphocytic infiltrates in the heart and liver (Fig. 2b, c) of mutant mice. 

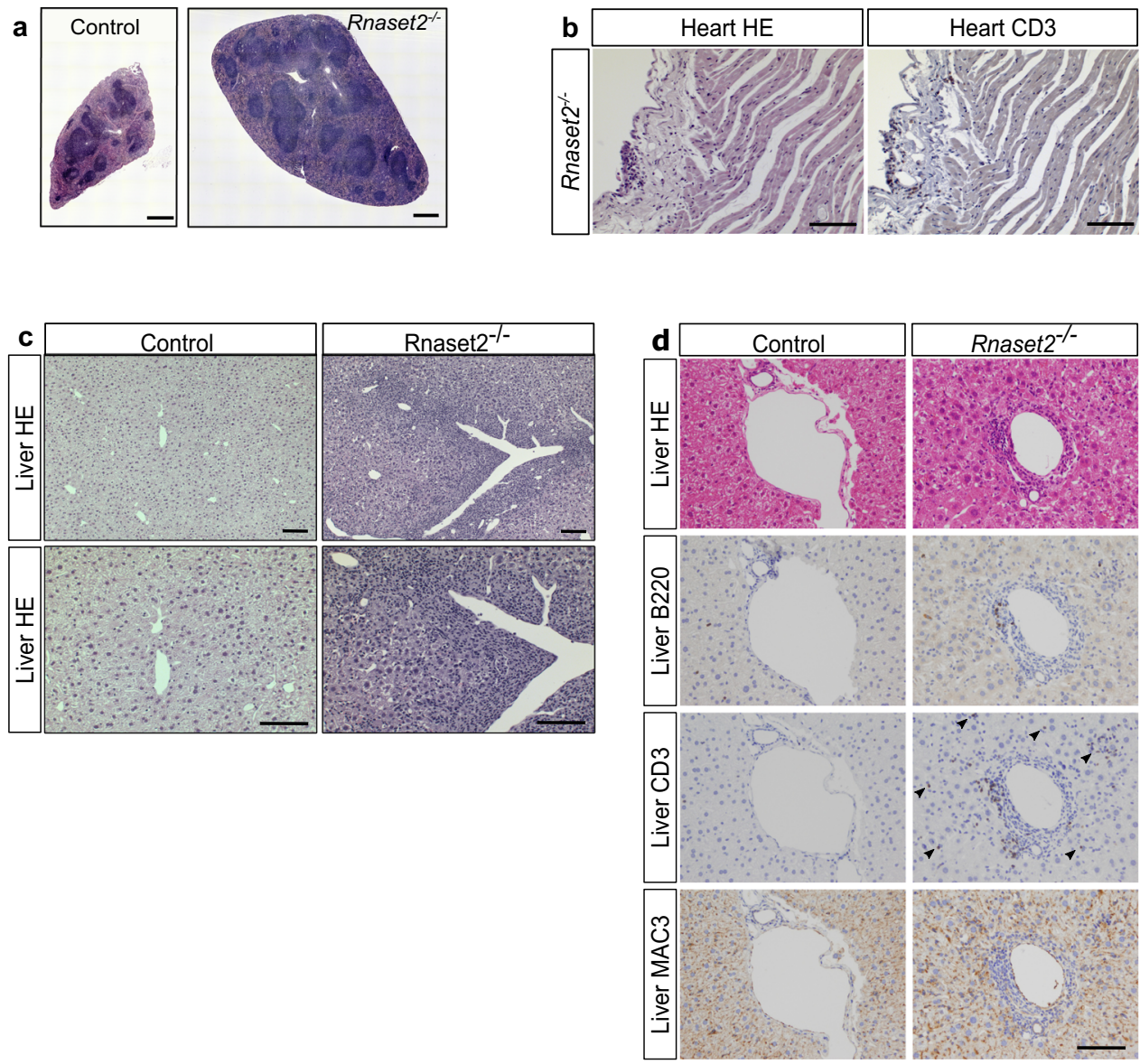

Fig. 2 Inflammatory organ disease. a Stitching of haematoxylin-eosin (HE) stained whole spleen cross section illustrates the enlargement and disorganisation of white splenic pulp in a 5-month-old mouse. $\times 20$ magnification. Scale bar, $500 \mu \mathrm{m}$. b HE staining of the heart showed lymphocytic infiltrates in the pericardium. Immunohistochemical staining revealed most of them as CD3 ${ }^{+} \mathrm{T}$ cells. Scale bar, $100 \mu \mathrm{m}$. $\mathbf{c ~ H i s t o l o g i c a l ~ H E ~ s t a i n i n g ~ o f ~ s l i d e s ~}$ from PFA-fixed and paraffin-embedded liver. Inflammatory lymphocytes infiltrate around the periportal fields pointing to a hepatitis. $\times 10$ and $\times 20$ magnification. Scale bar, $100 \mu \mathrm{m}$. d Immunohistochemical staining of periportal infiltrating cells revealed presence of B220 ${ }^{+} \mathrm{B}$ cells, CD3+ ${ }^{+}$cells and activated macrophages (MAC3 staining). Notice, T cells were spread over the whole liver parenchyma (arrowheads). $\times 20$ magnification. Scale bar, $100 \mu$ m. Images are representative of seven independent experiments.

Immunohistochemical staining identified $\mathrm{CD} 3^{+} \mathrm{T}$ cells, $\mathrm{B} 220^{+} \mathrm{B}$ cells and $\mathrm{MAC}^{+}$macrophages as the infiltrating cells in the periportal zone of the liver (Fig. 2d). T cells were found throughout the liver parenchyma (Fig. 2c, d). The inflammatory infiltrates in the pericardium of the heart also consisted of $\mathrm{CD}^{+}$ T cells (Fig. 2b).

Peripheral blood count and blood smears revealed leukocytosis and macrocytic hyperchromic anemia in mutant mice. Moreover, Rnaset $2^{-1-}$ mice showed reticulocytosis, illustrated by a higher number of blue stained enucleated erythrocytes compared to the control animals. Furthermore, severe thrombocytopenia was observed (Fig. 3a, b). Evaluation of bone marrow smears and immunohistochemical analysis of $\mathrm{CD}_{4} 1^{+}$megakaryocytes in spleen and bone marrow revealed increased numbers and morphological alterations (dysplasia) of megakaryocytes, demonstrated by single, small and round nuclei and megakaryocyte accumulation (Fig. $3 \mathrm{c}-\mathrm{e}$ ). In addition, Rnaset $2^{-1-}$ mice displayed significantly increased ALT values (185 \pm 109 vs. $33 \pm 9 \mathrm{U} / \mathrm{l})$, elevated hemolysis parameters (LDH $1624 \pm 676$ vs. $349 \pm 221 \mathrm{U} / 1$ and uric acid concentrations $6.0 \pm 1.2$ vs. $4.2 \pm 1.2 \mathrm{mg} / \mathrm{dl}$ ), while overall protein levels were decreased $(4.0 \pm 0.8$ vs. $5.0 \pm 0.3 \mathrm{~g} / \mathrm{dl})$ (Fig. 3f).

In line with its enlarged size, FACS analysis of spleen single cell suspensions (splenocytes) revealed a 5.1-fold increase in total cell count (Supplementary Fig. 2a). Multi-color flow cytometric analysis of hematopoietic precursors in the spleen demonstrated an elevated number of Ter119+ cells, indicating increased hematopoiesis of the erythroid lineage (Supplementary Fig. 2b). Specifically, we found an increased number of all HSC-dependent multipotent stem cells (common myeloid progenitor, granulocyte-macrophage progenitor and megakaryocyte-erythroid progenitor). In addition, all subsequent precursor cells of the myeloid and erythroid lineage were increased, with pre-megakaryocyte-erythroid (Pre-Meg-E) being the only exception (Supplementary Fig. 2c). In erythroid differentiation, we found a shift to immature erythroblasts (disproportional increase of R2 versus R3-R5) (Supplementary Fig. 2d, e).

Flow cytometric analysis of lymphocytes demonstrated that both absolute $\mathrm{CD}^{+}{ }^{+} \mathrm{T}$ cell and $\mathrm{CD}_{19}{ }^{+} \mathrm{B}$ cell numbers per spleen were significantly increased (Supplementary Fig. 3a). Likewise, the total numbers of both $\mathrm{CD}^{+}$and $\mathrm{CD}^{+} \mathrm{T}$ cells per spleen were significantly increased, and the CD4/CD8 ratio was significantly reduced (Supplementary Fig. $3 \mathrm{~b}$ ). Of note, the proportion of $\mathrm{CD}^{+}$ $\mathrm{T}$ cells harboring an effector memory phenotype was significantly increased while the proportion of naïve $\mathrm{CD}^{+} \mathrm{T}$ cells was significantly decreased (Supplementary Fig. 3c). The percentage and the absolute number of Ly6Chi CCR2 ${ }^{+}$monocytes were increased (Supplementary Fig. 3d).

Previous reports demonstrated an association of interferonopathies with autoimmunity and systemic lupus erythematosus in particular $12,31,32$. Consistent with other interferonopathies, we 

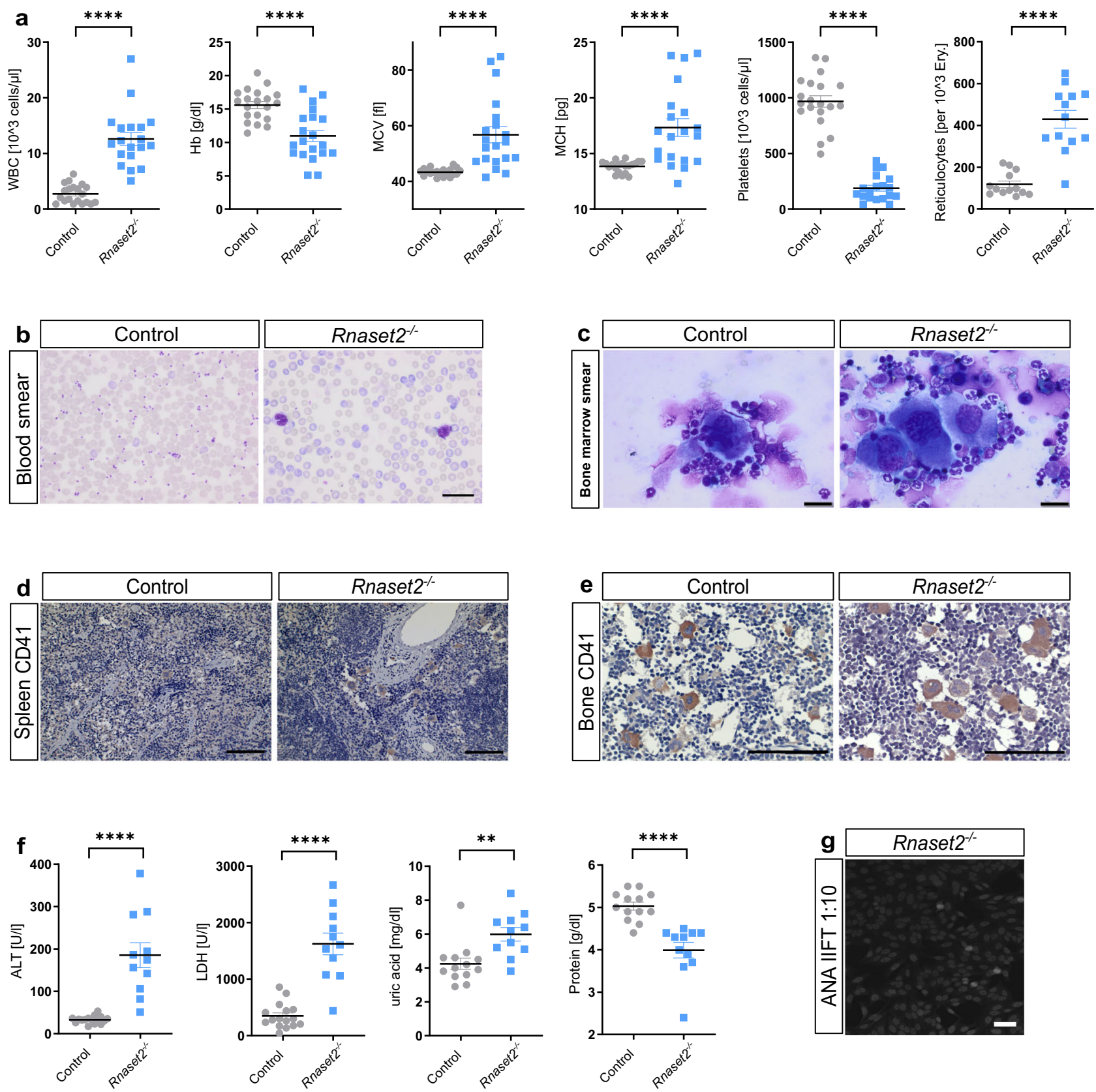

Fig. 3 Disturbed hematopoiesis and anti-nuclear antibody detection. a Blood counts from EDTA-stabilized whole blood of Rnaset2-/- and control mice revealed increased numbers of white blood cells (WBC, $n=19$ Rnaset2 ${ }^{-/-}$and $n=20$ control animals) and reticulocytes ( $n=13$ Rnaset2 $2^{-/-}$and control animals, respectively) in Rnaset2 ${ }^{-/-}$mice accompanied by highly significantly reduced platelets ( $n=19$ Rnaset $2^{-/}$-and $n=20$ control animals) and hemoglobin $(\mathrm{Hb})$ levels $\left(n=20\right.$ Rnaset2 ${ }^{-/-}$and control animals, respectively). The mean corpuscular volume (MCV) and mean corpuscular hemoglobin $(\mathrm{MCH})$ were significantly elevated $\left(n=20\right.$ Rnaset $^{-/-}$and control animals, respectively). Data depicted as the mean \pm SEM. $p$ values of two-tailed Student's $t$-test are represented as ${ }^{\star \star \star \star} p<0.0001$. b Blood smears of Rnaset $2^{-1-}$ and control mice clearly demonstrated the severe thrombocytopenia and the massive reticulocytosis (purple cells) in three biological replicates for control and Rnaset2 ${ }^{-/-}$, respectively. Scale bar, $25 \mu \mathrm{m}$. c Bone marrow smear and immunohistochemical staining of $\mathbf{d}$ spleen and $\mathbf{e}$ bone marrow showed a higher density of $\mathrm{CD} 41^{+}$megakaryocytes, which presented dysplastic features demonstrated by single, small, and round nuclei and megakaryocyte agglomerations. Spleen and bone marrow images are representative of three independent experiments, scale bar, $100 \mu \mathrm{m}$. Bone marrow smear images are representative of four biological replicates for control and Rnaset2 ${ }^{-/-}$, respectively, scale bar, $20 \mu \mathrm{m}$. $\mathbf{f}$ Depicted values of clinical chemistry from serum samples of Rnaset2 ${ }^{-/}-(n=11$ animals $)$ and control $(n=16$ animals for $\mathrm{ALT}$ and $\mathrm{LDH}$ and $n=13$ for uric acid and protein) mice were significantly altered, whereas further parameters measured remained unremarkable. Data depicted as the mean \pm SEM. $p$ values of two-tailed Student's $t$-test are represented as ${ }^{\star \star} p=0.0027,{ }^{\star \star \star \star} p<0.0001$. ALT alanine aminotransferase, LDH lactate dehydrogenase. $\mathbf{g}$ Representative fluorescence microscopy image with a positive antinuclear antibody (ANA) indirect immunofluorescence test (IIFT) of Rnaset2-/- mouse serum (12 weeks old) with a titre of 1:10. Further titration even revealed a titre of 1:1000 for autoantibodies present in this serum. Scale bar, $50 \mu \mathrm{m}$. $p$ values are represented as ${ }^{\star \star \star \star} p<0.0001,{ }^{\star \star \star} p<0.001,{ }^{\star \star} p<0.01,{ }^{\star} p<0.05$ and not significant $p \geq 0.05$. Source data are provided as a Source data file. 
found higher levels of anti-nuclear antibodies in the sera of Rnaset $2^{-1-}$ mice compared to controls (Fig. 3g). Anti-nuclear antibodies were already present at the age of 6 weeks but did not lead to immune complex-mediated nephritis during the observation period of 6 months (Supplementary Fig. 4).

\section{Rnaset $^{-1-}$ mice show a strong interferon-stimulated gene response in multiple organs. We next analyzed ISG transcripts by qRT-PCR in various organs of Rnaset $2^{-1-}$ mice. We observed the highest increase of ISG transcripts in the brain, where the mRNA-abundance of Isg15, Usp18 and Cxcl10 was elevated 21- fold, 23-fold, and 20-fold, respectively. Moreover, Ifi27 and Ddx58 were increased in the brain of Rnaset $2^{-1-}$ mice 3.4-fold and 6.44-fold, respectively (Supplementary Fig. 5a). In the liver, Ifi27 (8.2-fold), Rsad2 (6.8-fold), and Siglec1 (11.3-fold) were elevated (Supplementary Fig. 5b). In the spleen, Isg15 and Rsad2 were increased 9.4-fold while Ifi27 and Irf7 displayed about 2.5-fold increased mRNA-abundance (Supplementary Fig. 5c). Altogether, Rnaset $2^{-1-}$ mice display a strong interferon response in several organs, most prominently in the brain.}

Rnaset $2^{-1-}$ mice develop neuroinflammation and brain atrophy. Histology of Rnaset $2^{-1-}$ mouse brain and spinal cord revealed focal immune cell infiltration, including $\mathrm{CD}^{+} \mathrm{T}$ cells, $\mathrm{MAC}^{+}$macrophages/activated microglia, and polymorphonuclear granulocytes (Fig. 4a). CD3 ${ }^{+}$lymphocytes were predominantly $\mathrm{CD}^{+}$and presented in a perivascular and diffusely infiltrating manner (Fig. $4 \mathrm{a}, \mathrm{b}$ ). The focal inflammatory lesions were accompanied by monocyte infiltration and microglia activation and affected the grey and white matter of all brain regions and the spinal cord (Fig. 4c). In particular, cortex and hippocampus were affected by diffusely infiltrated $\mathrm{CD} 8^{+} \mathrm{T}$ cells (Fig. 4a). Myelin immunohistochemistry did not reveal any evidence for demyelination (Supplementary Fig. 6).

Flow cytometric ex vivo analysis of the CNS inflammatory infiltrates in Rnaset $2^{-/-}$mice was consistent with histopathology and demonstrated significantly increased numbers of $\mathrm{CD}^{+} \mathrm{T}$ cells $\left(\mathrm{CD} 45^{+} \mathrm{TCR}^{+} \mathrm{CD}^{+}\right)$and significant but numerically less impressive increases in $\mathrm{CD} 4^{+} \mathrm{T}$ cells $\left(\mathrm{CD} 45^{+} \mathrm{TCR} \beta^{+} \mathrm{CD} 4^{+}\right), \mathrm{B}$ cells $\left(\mathrm{CD} 45^{+} \mathrm{CD} 19^{+}\right)$and inflammatory monocytes $\left(\mathrm{CD} 45^{+}\right.$ $\mathrm{CD}_{11} \mathrm{~b}^{+}$Ly6C $^{\text {hi }} \mathrm{CCR}^{+}$) compared to controls (Fig. 4d). Of note, infiltrating $\mathrm{CD}^{+} \mathrm{T}$ cells predominantly harbored an effector memory phenotype $\left(\mathrm{CD} 45^{+} \mathrm{TCR} \beta^{+} \mathrm{CD} 8^{+} \mathrm{CD} 44^{\mathrm{hi}} \mathrm{CD} 62 \mathrm{~L}^{-}\right)$ (Fig. 4e and Supplementary Fig. 7).

The immunohistologically proven neuroinflammation in Rnaset $2^{-1-}$ mice was confirmed by contrast-enhanced cerebral magnetic resonance imaging (MRI) revealing significant leakage of the blood-brain barrier for gadopentetate-dimeglumine (GdDTPA) (Fig. 5a).

Volumetric MRI of the hippocampi at the age of 4-6 months demonstrated significant hippocampal atrophy in Rnaset $2^{-1-}$ mice compared to controls (Fig. 5b, c). A significant increase in $\mathrm{T}_{2}$ relaxation time in the periventricular region, hippocampus and cortex of Rnaset $2^{-1-}$ mice was observed compared to controls, a well accepted MRI marker for inflammation (Fig. 5d) ${ }^{33}$. Furthermore, ventricles of Rnaset $2^{-1-}$ mice were enlarged at 4-6 months (Fig. 5b, e), indicating brain volume loss.

Rnaset $^{-1-}$ mice exhibit learning difficulties. To assess whether neuroinflammation and neurodegeneration impair cognitive functions in Rnaset $2^{-1-}$ mice the cross maze task was employed to measure alternation frequency as an indicator of spatial working memory. Rnaset $2^{-1-}$ mice showed a trend towards reduced alternation rates $(31.7 \pm 11.8 \%$ vs. $39.2 \pm 9.7 \% ; p=0.061)$ at the age of 4 months. The total number of arm entries
$(24.8 \pm 10.3$ vs. $36.8 \pm 11.2 ; p<0.001)$ and the average distance $(16.0 \pm 4.9$ vs. $24.1 \pm 5.6 \mathrm{~m} ; p<0.001)$ were significantly decreased (Fig. 6a). Accordingly, the average speed in the open field paradigm was significantly reduced $(0.05 \pm 0.02$ vs. $0.03 \pm 0.01 \mathrm{~m} / \mathrm{s}$; $p<0.01$ ) (Fig. 6b). Basic motor performance, tested by balance beam and inverted grid hanging tasks, was not impaired (Supplementary Fig. 8a, b). Learning and memory were also assessed using the novel object recognition task (NOR). In general, mice without any cognitive impairment spend more time exploring a novel object compared to a familiar object. On day 1, when two identical objects were presented, Rnaset $2^{-1-}$ and control mice equally explored both objects (Fig. 6c). However, on the testing day (day 2), Rnaset $2^{-1}$ mice spent significantly less time exploring the novel object compared to control mice $(P<0.05$; Fig. 6d) and showed a general impairment in object discrimination, as also seen in the significantly lower discrimination ratio $(0.15 \pm 0.26$ vs. $0.39 \pm 0.11 ; p<0.01) \quad$ (Fig. 6e). The average exploration time for the two objects on day 1 and day 2 was the same for Rnaset $2^{-1-}$ and control mice (Fig. 6c, d).

Single nuclei RNA sequencing confirms type I interferon driven neuroinflammation and provides insights into the mechanism of cognitive impairment. To identify which cell types and pathways are involved in neuroinflammation we performed single nuclei RNA sequencing analysis of the caudate putamen (Fig. 7) and hippocampus (Supplementary Fig. 9). Clustering analysis revealed obvious differences when comparing Rnaset $^{-1-}$ to control tissue in the caudate putamen (Fig. 7a). Expression changes were particularly evident for a cluster that we identified as microglia/monocytes but also for astrocytes, oligodendrocytes, oligodendrocyte precursor cells (OPC), and three clusters representing neurons (neuronal cluster 2, 3, and 4; Fig. 7a). When we compared the differentially expressed genes amongst Rnaset $2^{-1-}$ and control mice across all cell types, we observed a strong upregulation of gene ontology (GO) biological processes linked to an immune response and specifically to type I interferon signaling (Fig. 7b, upper panel). The induction of the type I interferon-signaling was particularly obvious in the cluster that we had identified as microglia/monocytes and to a lesser extent in endothelial cells, oligodendrocytes, and OPCs (Fig. 7b, lower panel).

Therefore, we decided to analyze the microglia/monocyte cluster in more detail and observed that it consisted of microglia that were specific to either Rnaset $2^{-1-}$ or control mice (Fig. 7c). While popular microglia marker genes such as P2ry12, Tmem119, and Siglec-h were slightly decreased upon activation, expression of $\mathrm{C} 4 \mathrm{~b}$, an established marker for reactive microglia was highly upregulated in Rnaset $2^{-1-}$ mice (Fig. $\left.7 \mathrm{~d}\right)^{34}$. Two additional clusters were specific to Rnaset $2^{-1-}$ tissue and represented infiltrating monocytes and $\mathrm{CD}^{+} \mathrm{T}$ cells, as indicated by the expression of corresponding marker genes (Tbrc2, Cd8a, Gzmb and Stat4 for CD8 ${ }^{+} \mathrm{T}$ cells; Ccr2, Ly6c2, and CD74 for infiltrating monocytes) (Fig. 7d). In addition, a number of interferon-stimulated genes (Isg15, Usp18, Cxcl10, and Ddx58) previously determined in various organs by $\mathrm{qPCR}$ were also increased in microglia, infiltrating monocytes and $\mathrm{CD}^{+} \mathrm{T}$ cells of Rnaset $2^{-1-}$ mice (Fig. 7e).

To validate the single nuclei RNA sequencing results on the protein level, we carried out immunostainings of interferonstimulated gene 15 (ISG15) in the cortex of Rnaset $2^{-1-}$ and control mice. We found an impressive upregulation of ISG15 in various cell types in the cortex of Rnaset $2^{-1-}$ mice (Fig. 7f, left panel). Co-immunostaining revealed an upregulation of ISG15 particularly in astrocytes (GFAP), microglia (IBA1), and endothelial cells (CD31) (Fig. 7f, right panel). 


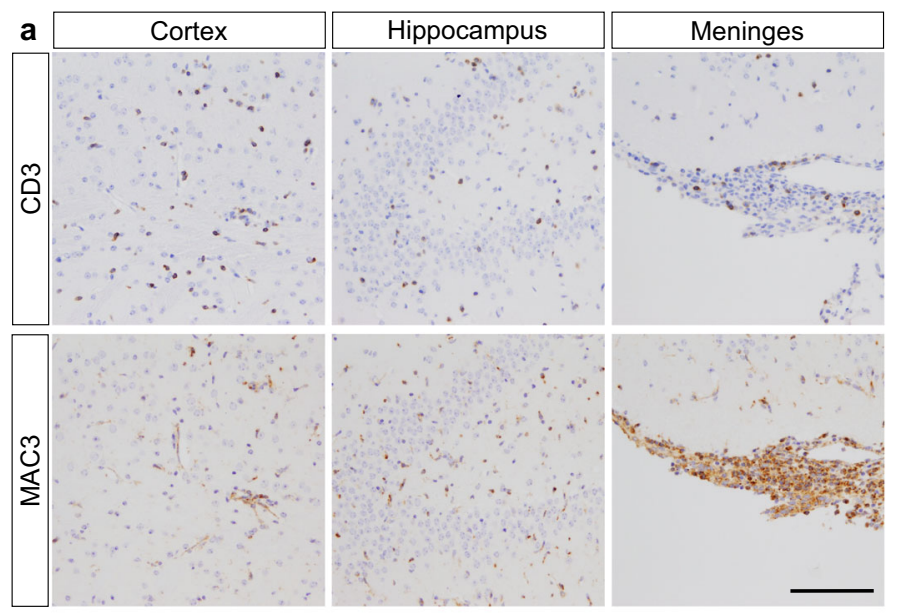

\begin{tabular}{|lccccc|} 
Animal & C & BS & Cb & SC & Mi \\
\hline KO-1 & 5 & 8 & 1 & 7 & + \\
\hline KO-2 & 11 & 2 & 1 & N/A & $+/-$ \\
\hline KO-3 & 6 & 3 & 0 & N/A & $+/-$ \\
\hline KO-4 & 16 & 4 & 5 & 2 & - \\
\hline KO-5 & 3 & 2 & 0 & 1 & - \\
\hline Control-1 & 0 & 0 & 0 & N/A & - \\
\hline Control-2 & 0 & 0 & 0 & N/A & - \\
\hline Control-3 & 0 & 0 & 0 & N/A & - \\
\hline Control-4 & 0 & 0 & 0 & 0 & - \\
\hline Control-5 & 0 & 0 & 0 & 0 & - \\
\hline
\end{tabular}
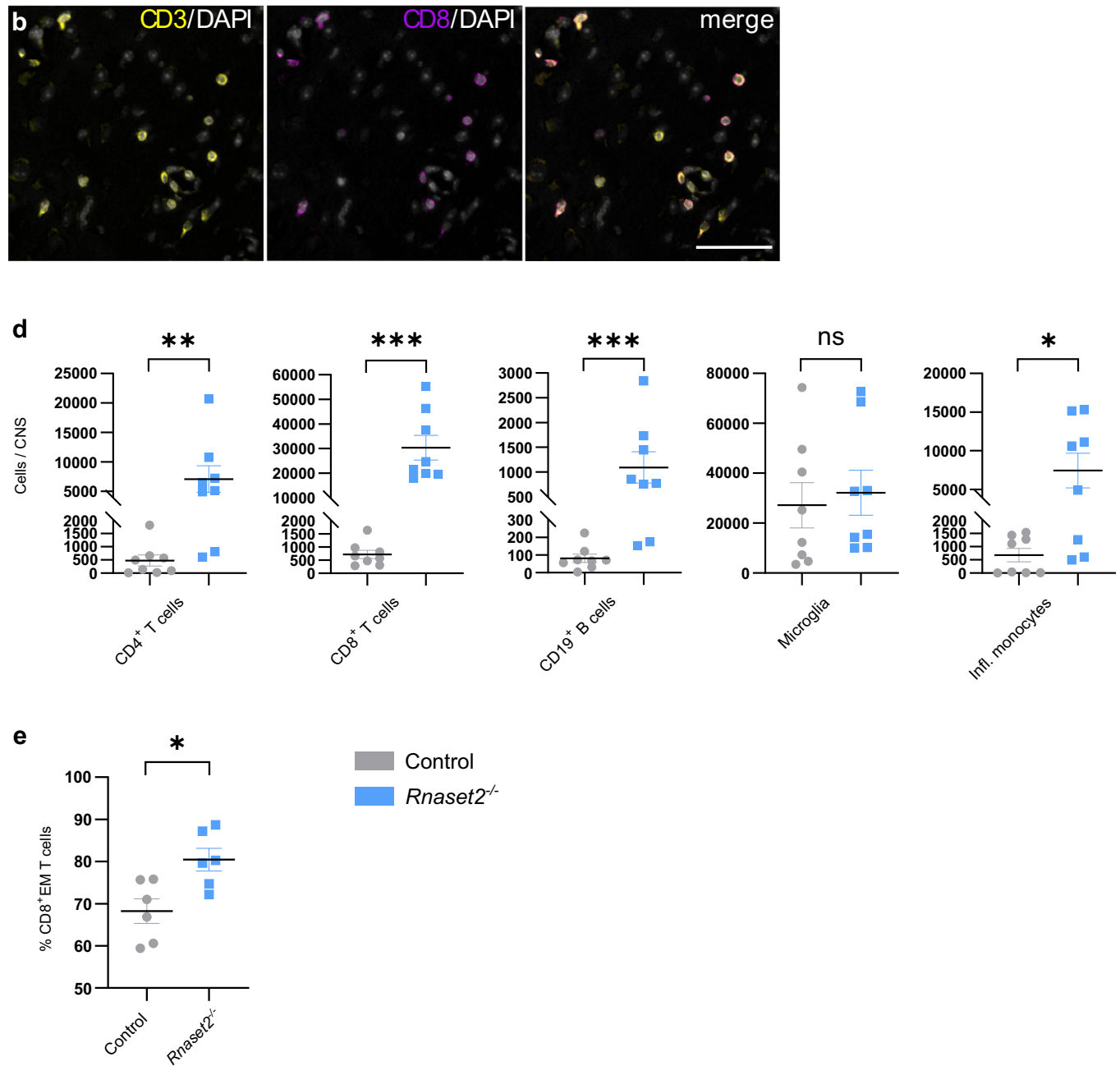

Control

Rnaset2-

We also analyzed the expression profiles of astrocytes, oligodendrocytes, and oligodendrocyte precursor cells in Rnaset $2^{-1-}$ and control mice (Supplementary Fig. 10). Astrocytes from Rnaset2 $2^{-1-}$ mice were characterized by a diminished processes associated with neuronal support functions such as ion transport (Supplementary Fig. 10b). GO-analysis furthermore revealed that oligodendrocytes from Rnaset $2^{-1-}$ mice contribute to interferon-mediated inflammation while processes linked to neuronal support such as axon development are reduced (Supplementary Fig. 10c). An induction of inflammation-related processes was also observed in oligodendrocyte precursor cells (Supplementary Fig. 10d).
In addition, we analyzed the neuronal clusters affected in Rnaset $2^{-1-}$ mice. Neuronal cluster 2 showed an obvious difference between Rnaset $2^{-1-}$ and control mice (Supplementary Fig. 10e). The specific expression of the marker genes Gad1 and Gad2 indicated that these cells mainly represented GABAergic inhibitory neurons (Supplementary Fig. 10f). Differential expression analysis revealed that processes related to energy metabolism and mitochondria function were decreased in this neuronal cluster, which is in line with data suggesting that loss of mitochondrial oxidative phosphorylation precedes neuronal degeneration (Supplementary Fig. 10g) ${ }^{35-37}$. Genes upregulated 
Fig. 4 Neuroinflammation. a Immunohistochemistry of T lymphocytes (CD3 positive, upper row) and macrophages/ activated microglia (MAC3 positive, lower row) in cortex, hippocampus and meninges of Rnaset2 $2^{-/}$mice revealed diffuse infiltration. Scale bar, $100 \mu \mathrm{m}$. $\mathbf{b}$ Co-immunostaining of $\mathrm{CD}^{+}$and $\mathrm{CD}^{+}$cells in white matter showed that most infiltrated T cells were CD8 positive. Scale bar, $50 \mu \mathrm{m}$. c Systematic evaluation of perivascular CD3 ${ }^{+}$cell infiltrations in standard slices from five Rnaset2-/- and control mice underline the involvement of the whole CNS. Of note, grey and white matter were involved in all regions. $\mathrm{C}=$ cerebrum, $\mathrm{BS}=$ brain stem, $\mathrm{Cb}=$ cerebellum, $\mathrm{SC}=$ spinal cord, $\mathrm{Mi}=$ meningeal inflammation, $\mathrm{N} / \mathrm{A}=$ not analyzed. Images in $\mathbf{a}$, $\mathbf{b}$ are representative of these five animals. $\mathbf{d}$ FACS analysis of total CNS (brain and spinal cord). Data depicted as the mean \pm SEM. $p$ values of two-tailed Mann-Whitney test and two-tailed Student's t-test (only for microglia) are represented as ${ }^{\star} p<0.05,{ }^{\star \star} p<0.01,{ }^{\star \star \star} p<0.001$ and not significant $p \geq 0.05$ of Rnaset2 ${ }^{-/-}(\mathrm{n}=8)$ and control mice $(\mathrm{n}=8)$. Rnaset2 ${ }^{-/-}$mice revealed significantly increased numbers of $\mathrm{CD} 4^{+} \mathrm{T}_{\text {-cells }}\left(\mathrm{CD} 45^{+} \mathrm{TCR} \beta^{+} \mathrm{CD} 4^{+}\right.$, $p=0.0019), \mathrm{CD} 8^{+}$T-cells $\left(\mathrm{CD} 45^{+} \mathrm{TCR} \beta^{+} \mathrm{CD} 8^{+}, p=0.0002\right), \mathrm{B}$ cells $\left(\mathrm{CD} 45^{+} \mathrm{CD} 19^{+}, p=0.0006\right)$ and inflammatory monocytes $\left(\mathrm{CD} 45^{+} \mathrm{CD} 11 \mathrm{~b}^{+}\right.$ Ly6Chi $^{\text {CCR2 }}{ }^{+}, p=0.0281$ ) whereas microglia cells (CD45 int CD11bint Ly6C - Ly6G ${ }^{-}$) were not significantly different $(p=0.7014)$; data pooled from three independent experiments. e Significant elevation $(p=0.0118)$ of $\mathrm{CD} 8^{+}$effector memory T cells $\left(\mathrm{CD} 45^{+} \mathrm{TCR}^{+} \mathrm{CD}^{+} \mathrm{CD}^{+} 4^{\text {hi }} \mathrm{CD} 62 \mathrm{~L}^{-}\right)$in $R$ Raset2 ${ }^{-/}$ mice by FACS analysis, $n=6$ Rnaset2 ${ }^{-/-}$and control mice; data pooled from three independent experiments. Data presented as mean \pm SEM. $p$ values of two-tailed Student's $t$-test is depicted ${ }^{*} p<0.05$. The corresponding gating strategy is depicted in Supplementary Fig. 7. Source data are provided as a Source data file.

in this neuronal cluster were mainly linked to a defense response to viruses and type I interferon signaling (Supplementary Fig. 10g). Nuclear transcriptional profiles of neurons related to cluster 3 and 4 in control mice were almost completely absent in Rnaset $2^{-1-}$ mice, indicating a prominent change in their transcriptional profiles. The specific expression of the marker genes Vglut1 and Vglut2 in cluster 3 suggest that these cells are excitatory glutamatergic neurons, while neuronal cluster 4 consists of parvalbumin positive GABAergic inhibitory neurons (Supplementary Fig. 10f).

A similar analysis of cluster-specific gene ontology (GO) biological processes was carried out for hippocampal tissue (Supplementary Fig. 9). We observed a strong inflammatory response driven primarily by microglia cells (Supplementary Fig. 9a,b). In the hippocampus we saw the same activation of microglia compared to caudate putamen, which is reflected by a decreased expression of popular microglia genes (P2ry12 and Siglec- $h$ ) and the expression of the reactive microglia marker $\mathrm{C} 4 \mathrm{~b}$. The upregulation of interferon-stimulated genes (Isg15, Usp18, Cxcl10, and $D d x 58)$ was also present in hippocampal microglia cells of Rnaset $2^{-1-}$ mice (Supplementary Fig. 9c). Although few genes were differentially expressed in oligodendrocytes and OPCs, there was a strong induction of the Dddx60 gene, which codes for an RNA helicase that promotes RIG-I, MDA5, and LGP2-mediated induction of interferon-signaling (Supplementary Fig. 9d) ${ }^{38}$. These inflammatory responses were associated with the loss of a neuronal cluster representing GABAergic inhibitory neurons and a severe dysregulation of the synaptic plasticity genes in excitatory glutamatergic neurons (Supplementary Fig. 9e,f).

Inflammation and in particular type I interferons are known to increase major histocompatibility complex (MHC) class I expression on many cells ${ }^{39,40}$. Not surprisingly, the MHC class I genes $H 2-D 1, H 2-K 1$, and $B 2 M$ were among the top regulated genes in our nuclear RNA sequencing data set and their expression was significantly increased in oligodendrocytes, OPCs, astrocytes, neurons, and microglia (Supplementary Fig. 11a-f). To verify these transcriptional findings, we analyzed the MHC class I expression on microglia cells by flow cytometry, which was significantly increased in Rnaset $2^{-1-}$ compared to control mice (Supplementary Fig. 11g).

In summary, single nuclei RNA sequencing was able to identify type I interferon signaling in all CNS cell types. Furthermore, we found evidence for the dysregulation of homeostatic functions in astrocytes, oligodendrocytes and OPCs along with marked alterations in neuronal subclusters.

Neuroinflammation and hippocampal atrophy in Rnaset ${ }^{-1-}$ mice are IFNAR1-mediated. To investigate whether the CNS inflammation observed in Rnaset $2^{-1-}$ mice is indeed type I interferon-mediated, Rnaset $2^{-1-}$ mice were crossbred to Ifnar $1^{-1-}$ mice. Rnaset $2^{-1-}$ Ifnar $1^{-1-}$ and age-matched Rnaset $2^{-1-}$ mice, as well as control mice, were sacrificed at month six, and brain tissue was collected for immunohistochemical analysis (Fig. 8a). Whereas the Rnaset $2^{-1-}$ mice presented with the typical foci of mononuclear infiltration consisting of $\mathrm{T}$ cells and monocytes in grey and white matter regions, the Rnaset $2^{-/}$Ifnar $1^{-1-}$ and control mice did not show any infiltration of peripheral immune cells into the brain (Fig. 8a,b). Furthermore, microglia activation was not observed in Rnaset $2^{-1-}$ Ifnar1 $1^{-1-}$ and control mice (Fig. 8a). Importantly, inflammatory foci in Rnaset $2^{-1-}$ mice were accompanied by upregulation of ISG15, which was entirely absent in Rnaset2-/Ifnar $1^{-1-}$ and control mice as demonstrated by the color deconvolution of the DAB-ISG15 signal in slide images of the whole hemispheres (Fig. 8a and Supplementary Fig. 13). These data indicate that the concurrent lack of functional type I interferon signaling completely abrogates the neuroinflammatory phenotype elicited in Rnaset2-1- mice.

In line, the $\mathrm{T}_{2}$ relaxation time in the ex vivo MRI normalized in all analyzed brain areas including the hippocampus (Fig. 8c) and the ventricular enlargement disappeared. Of note, the volumetric analysis of the relative hippocampal area showed no evidence for hippocampal atrophy in Rnaset $2^{-1}$ - Ifnar $1^{-1-}$ mice (Fig. 8d, e).

Abolished type I interferon signaling in Rnaset $2^{-1-}$ Ifnar $1^{-1-}$ animals also reduced the observed organ enlargements of Rnaset $2^{-1-}$ mice and normalized many blood values (Supplementary Fig. 12a-c). In this regard, thrombocytopenia and leukocytosis improved but remained significantly different in Rnaset $2^{-1-}$ Ifnar $1^{-1-}$ animals compared to controls whereas the macrocytic hyperchromic anemia completely resolved (Supplementary Fig. 12c).

Importantly, the lack of IFNAR1 signaling in Rnaset $2^{-1-}$ Ifnar1 $1^{-I}$ mice also rescued the limited life expectancy of Rnaset $2^{-1-}$ mice (Supplementary Fig. 12d). Taken together, we provide compelling evidence that interferon-signaling is essential for neuroinflammation and neurodegeneration in Rnaset $2^{-1}$ mice and has a significant impact on the reduced life expectancy in this animal model.

\section{Discussion}

The pathophysiological understanding of congenital viral brain infections and inborn type I interferon-driven diseases has been impaired by the failure of many animal models to reproduce the neurological phenotype, which is a fundamental characteristic of these disorders. In this study, we characterize the newly generated Rnaset $2^{-1-}$ mice, in which the CNS, along with other organs, is prominently affected, and reveal that neuroinflammation and neurodegeneration strongly depend on IFNAR1-signalling. 
a
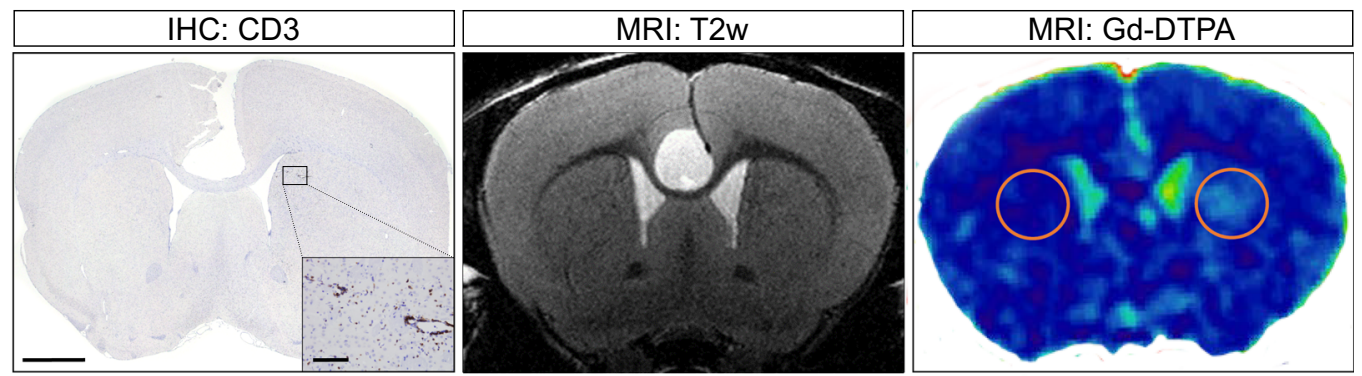

b
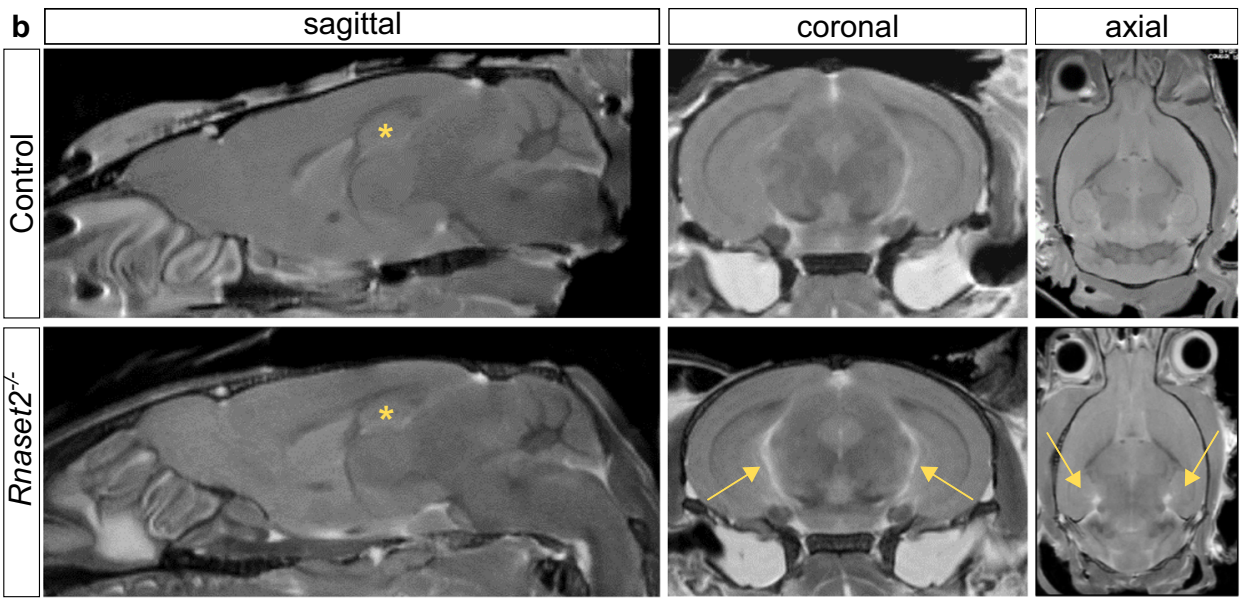

C
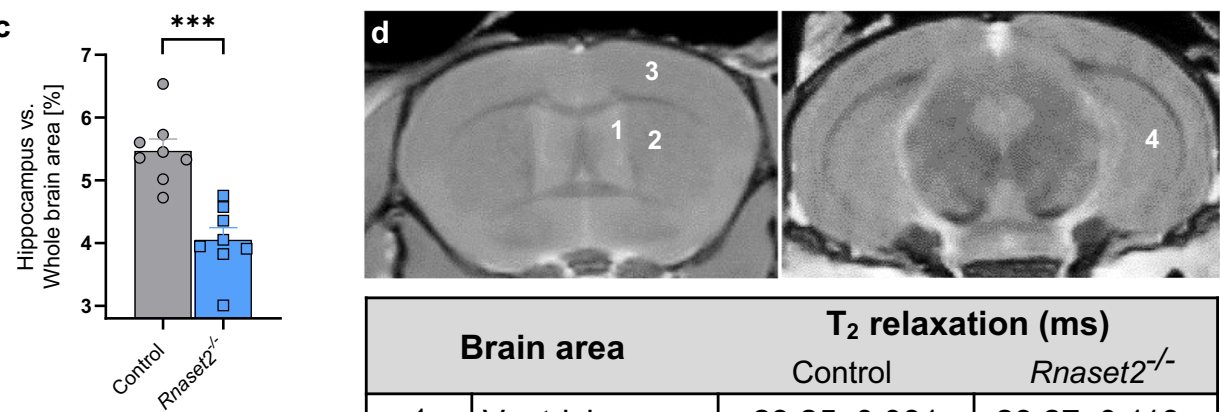

\begin{tabular}{|c|l|c|c|}
\hline \multicolumn{2}{|c}{ Brain area } & \multicolumn{2}{c|}{$\mathbf{T}_{2}$ relaxation (ms) } \\
Control & Rnaset2 ${ }^{-/-}$ \\
\hline 1 & Ventricle & $23.25 \pm 0.081$ & $23.27 \pm 0.118$ \\
\hline 2 & Periventricular & $21.64 \pm 0.241$ & $22.28 \pm 0.255^{*}$ \\
\hline 3 & Cortex & $21.12 \pm 0.415$ & $21.91 \pm 0.296^{*}$ \\
\hline 4 & Hippocampus & $22.06 \pm 0.452$ & $23.02 \pm 0.683^{*}$ \\
\hline
\end{tabular}
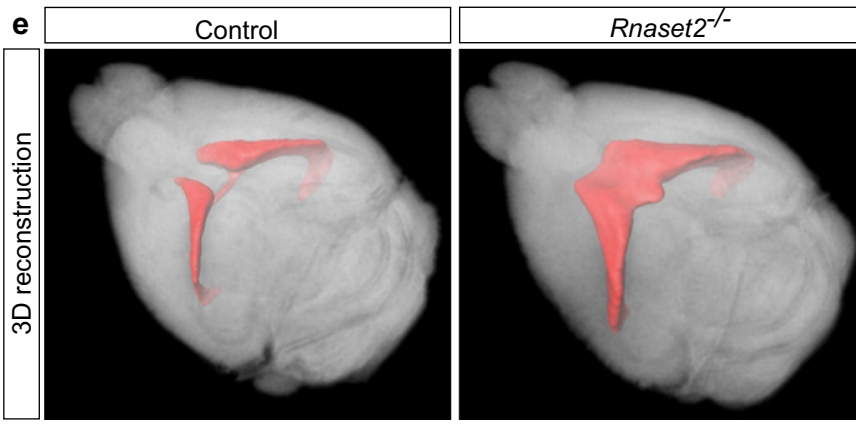

Rnaset ${ }^{-1-}$ mice develop a severe type I interferon-driven neuroinflammatory disorder, which is characterized by elevated ISGs, perivascular infiltrates of inflammatory monocytes, activated microglia, and diffuse infiltration of $\mathrm{CD}^{+} \mathrm{T}$ cells of the effector memory type throughout the entire CNS. The perivascular inflammation leads to blood-brain barrier leakage as shown by gadolinium enhancement on cerebral MRI. Furthermore, the observed neuroinflammation might foster the documented general brain atrophy, which is accentuated in the hippocampus. Not surprisingly, memory function is impaired in these mice.

The first indications of harmful effects of type I interferon on the brain were found in transgenic mice, in which astrocytes 
Fig. 5 Magnetic resonance imaging (MRI) of neuroinflammation and neurodegeneration. a Light microscopic image of CD3 immunohistochemistry (IHC: CD3) revealed a perivascular $T$ cell infiltration near the left ventricle (inlay, left image). Scale bar, $1 \mathrm{~mm}$ and $100 \mu \mathrm{m}$ respectively. Corresponding T2weighted coronary MRI (MRI: T2w) showed no remarkable alterations (middle image). Corresponding map of percentage signal enhancement (MRI: GdDTPA) revealed gadolinium enhancement in the region of perivascular T cell infiltration near the left ventricle in Rnaset2 $2^{-/-}$mice (red circles, right image). Representative images of $n=2$ biological replicates for Rnaset $2^{-/-}$and control mice, respectively. b Representative magnetic resonance anatomical images (of $n=8$ Rnaset2 ${ }^{-/}$and control mice, respectively) in sagittal, coronal, and axial plane showing significant hippocampal atrophy (asterisk) in Rnaset2 ${ }^{-/}$mice compared to controls. Ventricular enlargement, which may have been arisen due to shrinkage of hippocampus are shown with (arrows). c Percent area of hippocampus (left and right) with respect to whole brain area. The size of the hippocampus relative to the whole brain was significantly smaller $(p=0.0002) . n=8$ Rnaset2 ${ }^{-/}-$and control mice from two independent experiments. Data depicted as the mean \pm SEM. $P$ value of one-way ANOVA are presented as ${ }^{\star \star \star} p<0.001$. $\mathbf{d} \mathrm{T}_{2}$ relaxation time measurement in various brain regions displayed a significant increase in cortex, hippocampus and periventricular region of Rnaset2 ${ }^{-/-}$mice in $n=8$ Rnaset2 ${ }^{-/}$and control mice, respectively. Region of interest (ROI) selected for $T_{2}$ relaxation time measurements are shown in left images. ROI: (1) ventricle, (2) periventricular, (3) cortex, (4) hippocampus. Data depicted as the mean \pm SEM. $p$ values of one-way ANOVA are represented as ${ }^{\star} p<0.05$. e 3-dimensional reconstruction of the ventricular system in MRI showed an expansion in Rnaset2-/- (right side) versus control mice (left side) as an indication of a general brain atrophy. Source data are provided as a Source data file.

chronically secrete IFN-I ${ }^{8,9}$. The reported phenotype, including inflammatory angiopathy with mononuclear cell cuffing, brain atrophy, particularly of the hippocampus, and memory dysfunction resembles the phenotype of the Rnaset $2^{-1-}$ mouse described here. RNaseT2-deficient rats developed a more limited phenotype characterized by reactive astrocytes in the hippocampus and learning difficulties. These rats also showed indications of altered lysosomal function, but lacked cellular evidence of neuroinflammation and type I interferon signatures ${ }^{41}$.

The expansion and persistence of effector memory CD8 ${ }^{+}$ $\mathrm{T}$ cells is associated with both viral infection and $\mathrm{AGS}^{42-44}$. Moreover, in the CNS, adaptive immune cells and clonally expanded effector memory $\mathrm{CD} 8^{+} \mathrm{T}$ cells have come into focus in the pathogenesis of aging and Alzheimer's disease ${ }^{45,46}$. The observed neuroinflammatory response in Rnaset $2^{-/-}$mice with considerable numbers of $\mathrm{CD}^{+} \mathrm{T}$ cells exceeds the bystander effects observed in many neurodegenerative disease models ${ }^{47,48}$. It is tempting to speculate that the here reported increase in MHC class I expression on all CNS cells might support antigen-specific $\mathrm{CD}^{+} \mathrm{T}$ cell expansion in the CNS. Thus, clarifying if $\mathrm{CD} 8^{+}$ $\mathrm{T}$ cells are clonally expanded in the CNS of Rnaset $2^{-1-}$ mice and whether they participate in perpetuating neuroinflammation and neurodegeneration will be an important issue to be addressed in future studies. Understanding the extent to which $\mathrm{CD} 8^{+} \mathrm{T}$ cells contribute to disease pathogenesis is of great importance and with broad implications for neuroinflammatory and neurodegenerative diseases in general.

As shown by single nuclei RNA sequencing, type I interferon signaling in the CNS, is accompanied by a homeostatic dysfunction of glial cells (microglia, astrocytes, oligodendrocytes, and OPCs) and neurons. In order to improve the mechanistic understanding of type I interferon-driven neuropathological changes, we need to better understand how the observed transcriptional disturbances in glial cells and in neuronal subclusters contribute to the disease pathogenesis.

The unique inflammatory CNS phenotype in Rnaset $2^{-1-}$ mice requires 4-5 months to develop. The lack of CNS inflammation in many AGS mutants described so far might therefore be attributed to their premature death, either embryonically as in Dnase $2 a$ mutants or within days postnatally as in Adarl and Rnaseh $2 b / c$ mutants ${ }^{49}$. Alternatively, AGS-related genes might have more redundant or dispensable functions in mice than in humans. Rnaset $2^{-1-}$ mice share the described peripheral phenotype with many other AGS mouse models. Interferon signatures in various organs and a reduced survival have been described for Trex1, Adar1, and Ifih1 mutants $25,27,50$. Furthermore, $\mathrm{T}$ cells have been shown to infiltrate the heart of Trex1deficient mice, hepatocyte-specific Adar1-deficient mice and in gain-of-function mutation Ifih1 mutant mice respectively ${ }^{25,27,51}$.
In the hepatocyte-specific Adar1-deficient mice the infiltrating $\mathrm{T}$ cells are also predominantly of the effector memory subtype, suggesting that the clonal expansion of $\mathrm{T}$ cells could take place in the effector organ ${ }^{51}$. Rnaset $2^{-1-}$ mice share the disturbance of hematopoiesis, a prominent feature of AGS mouse models, with Trex1, Adar1, and Dnase $2 a$ mutants ${ }^{26,49,52}$. The anemia of Rnaset $2^{-1-}$ mice might recapitulate the hematopoietic disorder of erythroid-restricted deletion of $A d a r 1^{52}$. We found increased ANA autoantibodies in Rnaset $2^{-1-}$ mice, but no evidence for kidney pathology. This is different from Ifih1 mutants, which develop an antibody-mediated lupus-like nephritis, or from Trex1-deficient mice, which develop lupus-like inflammation in multiple organs ${ }^{25,44}$.

IFNAR1-deficiency in Rnaset $2^{-1-}$ mice strikingly abrogates the CNS inflammation observed in Rnaset $2^{-/-}$IFNAR1-competent mice and prolongs their life expectancy. However, not all perturbations are completely abolished, and hepatosplenomegaly, thrombocytopenia, and leukocytosis are only ameliorated. Thus, future therapeutic approaches against interferonopathies should not only take into account the underlying mechanisms of how nucleic acids activate the interferon-signaling pathway but also the processes that lead to tissue-specific effects of IFN-I. In this regard, we and others unraveled the interplay of TLR8 and RNaseT2 recently. Human RNaseT2 and RNase2 act synergistically to degrade longer, structured RNA molecules into TLR8 ligands. Thus, cells from RNaseT2 hypomorphic patients demonstrated reduced activation of TLR $8^{21,22}$. However, one can assume that type I interferon signaling is not the result of this reduced TLR8 activity, but rather the accumulation of longer RNA molecules that activate other RNA-binding PRRs and trigger interferon production ${ }^{53}$. This would be in line with what has been observed for another endolysosomal nuclease, DNase2, which both contributes to TLR9 activation and inhibits activation of the cytosolic DNA receptors cGAS and AIM2 $2^{53-56}$. In addition to signaling PRRs, viral restriction factors, such as PKR and OAS1, are ISGs and also sense cytosolic RNA, leading to substantial cell-intrinsic effects. Given that the immune sensing mechanisms activating the interferon pathway are also activated by viral nucleic acids, it is not surprising that the human CNS phenotype of RNaseT2-deficient CLE recapitulates congenital viral infection ${ }^{1}$. However, further studies will be necessary to assess which of the candidate anti-viral RNA receptors in fact contribute to this phenotype.

Altogether, the Rnaset $2^{-/-}$mouse is an important addition to the repertoire of mouse models for type I interferonopathies. With its unique ability to recapitulate the human neuroinflammatory phenotype of RNaseT2-deficiency, it will provide important insights into the neurological pathomechanisms of human RNaseT2 deficiency. A better understanding of the 

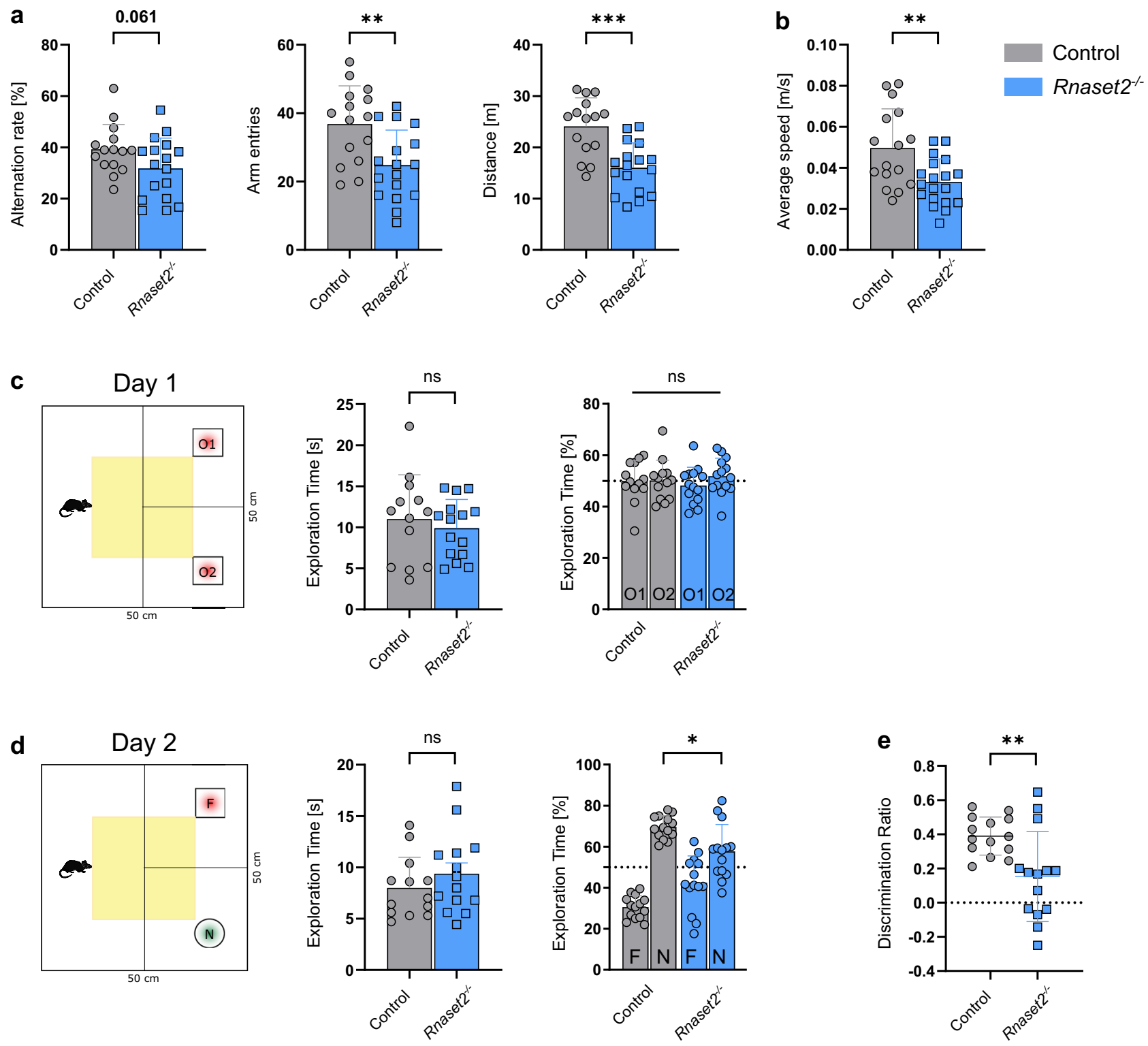

Fig. 6 Memory impairment in 4-month-old Rnaset2 $^{-/}$- mice. a Cross maze testing: Percentage of spontaneous alternation ( $\left.p=0.061\right)$, total arm entries, and the average distance in meters of Rnaset2 $2^{-/-}$(blue) and control mice (gray). Rnaset $2^{-/}-$mice showed less activity, documented by a reduced number of arm entries $(p=0.0034)$ combined with a significant decrease of the average distance $(p=0.0001)$ covered in the cross maze. $\mathbf{b}$ Graph depicting the significantly reduced average speed of Rnaset2 ${ }^{-/}$compared to control mice in the open field box $(p=0.0031)$. c Novel object recognition (NOR) analysis in an open field box. Mean percentage of time spent exploring two similar objects ( $\mathrm{O} 1$ and $\mathrm{O} 2$, red) and total exploration time on training day (Day 1 ) were similar in both genotypes. d Mean percentage of time spent exploring two different objects, the familiar ( $F$, red) and a novel one ( $N$, green), on testing day (Day 2) of the NOR test was significantly different between Rnaset2 ${ }^{-/}$mice and controls $(p=0.0456)$. e Discrimination ratio of Rnaset2 ${ }^{-/}$mice was significantly diminished $(p=0.0046)$. Rnaset2 ${ }^{-/-} n=17$, control $n=15$. Values are given as the mean \pm SEM. Statistical analysis was performed with unpaired t-test and with two-way ANOVA followed by Tukey's multiple comparison test. $p$ values are represented as ${ }^{\star \star \star \star} p<0.0001$, ${ }^{\star \star \star} p<0.001$, ${ }^{\star *} p<0.01,{ }^{\star} p<0.05$ and not significant (ns) $p \geq 0.05$. Source data are provided as a Source data file.

functional consequences of type I interferon-triggered pathways within the CNS will have far-reaching implications not only for inborn RNaseT2-deficient CLE and AGS but also for congenital viral infections. As such, it may be useful to facilitate the design of therapeutic approaches for these severe early childhood onset diseases.

\section{Methods}

Generation of RNaseT2 knockout mice. Embryonic stem cells (ES) from a JM8A3.N1 background harboring an engineered allele of the Rnaset2a gene were acquired from the European Conditional Mouse Mutagenesis Program

(EUCOMM). Chromosomal integration of the transgenic cassette deletes exon 2, 3 , and 4 of Rnaset2a [NM_001083938.3] leading to a frameshift and a translational stop in exon 5. ES were microinjected into blastocysts derived from C57BL/6N mice, and embryos were transferred to pseudo-pregnant NMRI foster mothers, yielding 10 chimeric males. For ES clone EPD0405_5_D12, germline transmission was achieved upon breeding with C57BL/6N females, yielding C57BL/6N Rnase$t 2 \mathrm{a}^{\mathrm{tm} 1(\mathrm{KOMP}) \mathrm{Wtsi}}(\mathrm{RNT} 2 \mathrm{~A}-\mathrm{KO})$ mice, which were maintained on a C57BL/6 N background. To create the Rnaset $2 a$ and $b$ double knockout strain C57BL/6 N

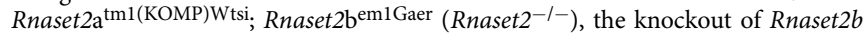
was generated on the background of RNT2A-KO strain by the CRISPR/Cas9 method $^{57}$. We injected the Cas9 protein instead of Cas9 coding plasmid DNA to reduce off-target effects ${ }^{58}$. The CRISPR/Cas9 target sequence was located in exon 2 [5'- ACAGUAUGCAAGGUGAG -3'; crRNA] of the Rnaset $2 b$ gene

[NM_026611.2] and identified with CRISPR guide design tool from the Massachusetts Institute of Technology (http://crispr.mit.edu:8079). 
a

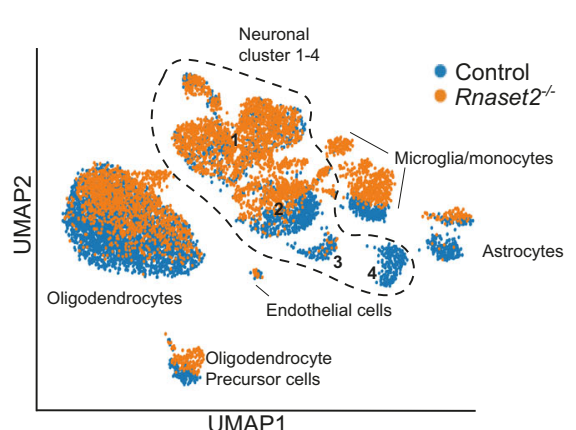

b
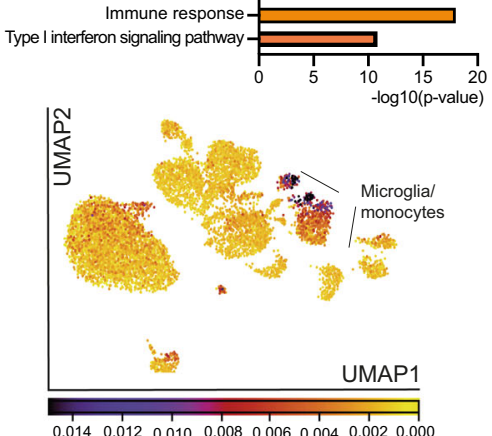

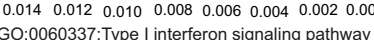
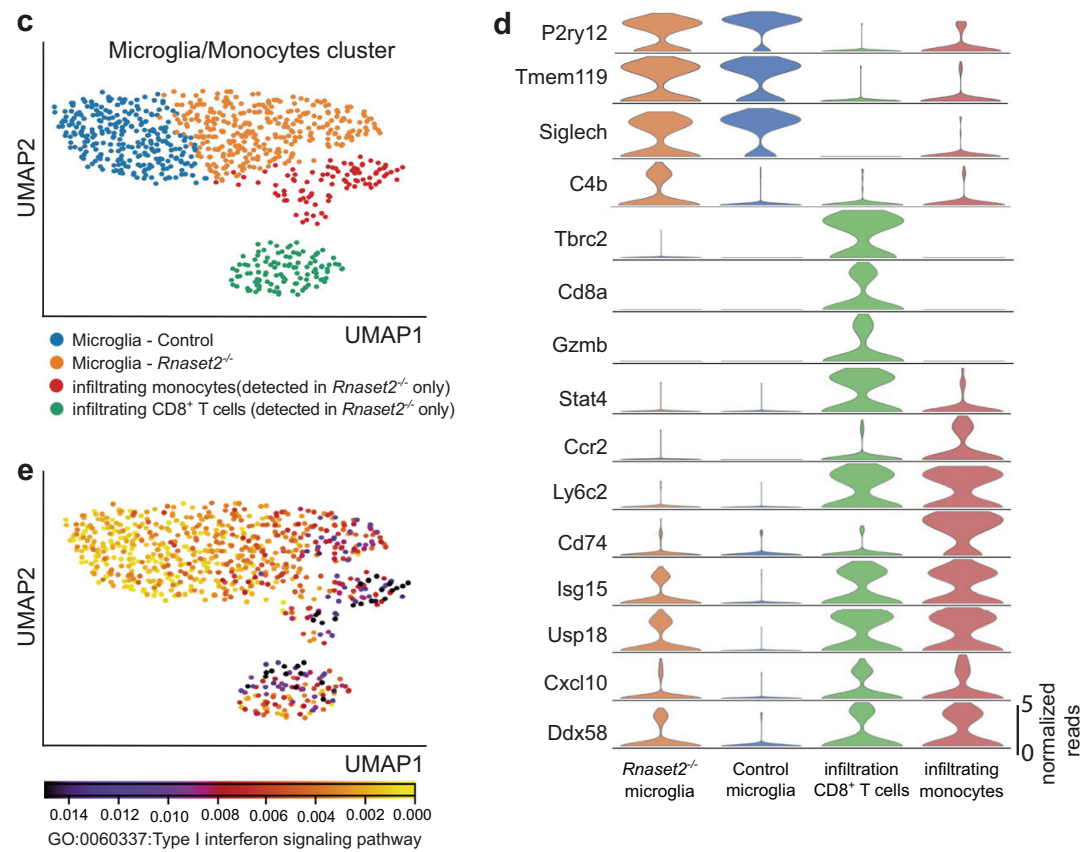

microglia microglia $\mathrm{CD}^{+} T$ cells monocytes

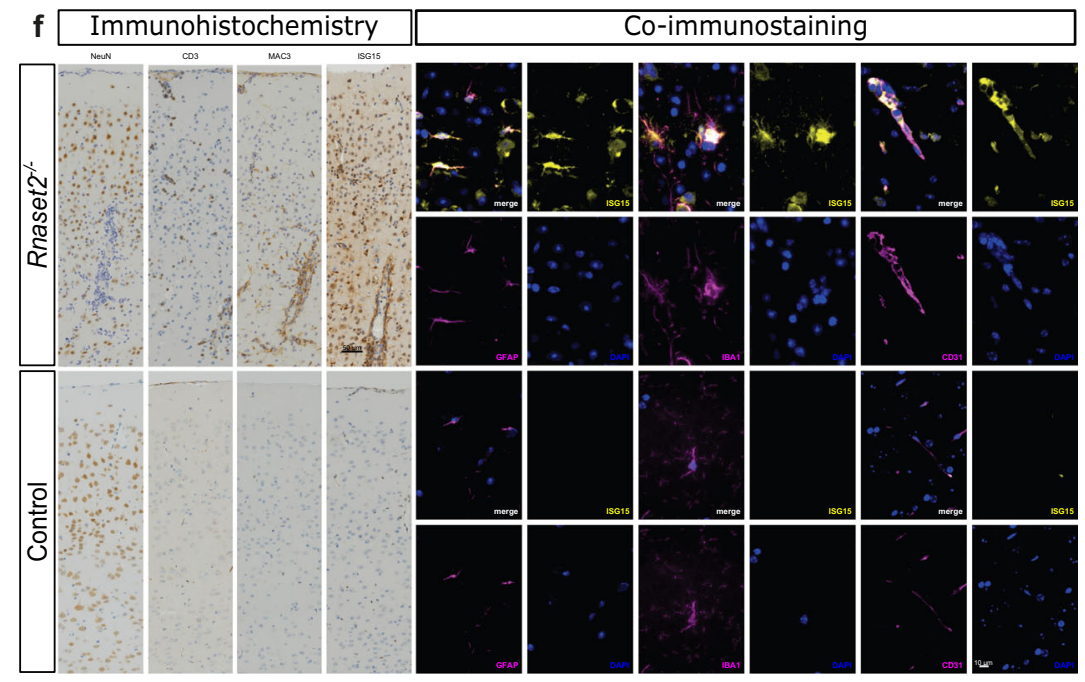

For Ribonucleoprotein- (RNP) mediated genome editing, zygotes of homozygous RNT2A-KO were microinjected with crRNA:tracrRNA:Cas9 RNPcomplex (Integrated DNA Technologies Inc., IDT)). In brief, $1.5 \mu \mathrm{M}$ crRNA was mixed with equimolar tracrRNA to allow the formation of the guideRNA. This was then added to $0.4 \mu \mathrm{M}$ S.p. Cas9 protein (IDT) to form the RNP-complex. A singlestranded template DNA (IDT) was added at last to this injection mix for homology directed repair (HDR) [5'- TTTTGTCACTTTAAAACAGTATTGAAAAATCAAT
TTTTCTTTTAACAGTGGCAGCCATGAGTGGAAAAAACTAATTTTGACCCA GCACTGGTAAGGATCCACAGTATGCAAGGTGAGCTCTGCTTTGACTGGC CCA $\left.-3^{\prime}\right]$. The resulting edit in the genome had an insertion of the translational stop codon TAA and a BamHI restriction site in exon 2 of Rnaset $2 b$ (c.145_147delinsTAA; p.Pro49Ter).

Routine genotyping of the Rnaset2a knockout allele was performed by genomic polymerase chain reaction (PCR) using primers P1 (5'-TACTTGCAGAAGGCCA 
Fig. 7 Single nuclei RNA sequencing analyses of the caudate putamen confirms type I interferon-driven neuroinflammation. a UMAP clustering of the caudate putamen from Rnaset $2^{-/}$and control mice. $\mathbf{b}$ Upper panel: Differential gene-expression was performed to compare Rnaset2 ${ }^{-/-}$and control mice across all detected cell types. GO-term analysis (biological processes) reveals immune response and type I interferon signaling as the major biological processes to be affected in the caudate putamen from Rnaset2-/- mice. Lower panel: UMAP indicating that the expression of the type I interferon signaling pathway (GO:0060337) is particularly obvious in the microglia/monocytes cluster. c UMAP clustering of the microglia/monocytes cluster clearly distinguished WT and Rnaset2-/- microglia and identifies infiltrating monocytes and CD8 ${ }^{+} \mathrm{T}$ cells. $\mathbf{d}$ Violin plot showing the expression of marker genes within the 4 different clusters detected in (C). e UMAP clustering of the microglia/monocytes cluster indicating that the expression of the type I interferon signaling pathway (GO:0060337) is particularly obvious in the microglia from Rnaset2 $2^{-/}$mice and within the infiltrating monocytes and $\mathrm{CD}^{+} \mathrm{T}$ cells. f Columns 1-4: IHC for neurons (NeuN), T cells (CD3), macrophages/activated microglia (MAC3) and ISG15 in the cortex of Rnaset2-/- (representative of $n=5$; upper panel) and control (representative of $n=6$; lower panel) mice demonstrates the diffuse and perivascular infiltration of T cells (CD3) and macrophages/activated microglia (MAC3) and an extensive expression of ISG15 in Rnaset2-/- mice. Scale bar, 50 $\mu$ M. Columns 5-10: Exemplary coimmunofluorescence staining confirm expression of ISG15 in astrocytes (GFAP), microglia/macrophages (IBA1), and endothelial cells (CD31) in

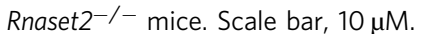

GAAGTGGG-3'), P2 (5'-CGCTTTTCTGGATTCA-TCGACTGTGG-3'), and P3 (5'-GACCCAGGGGCTCAGTACTAGTG-3') producing a product of $838 \mathrm{bp}$ for WT Rnaset2a allele and 870 bp for the transgene. For Rnaset $2 b$-HDR allele genotyping was performed by genomic PCR with primers P4 (5'-CTGTCCTCTGT GGTGTGTATG-3') and P5 (5'-TGGAAGTATACCC-TCTCTCGGC-3') followed by Sanger sequencing with the same primers.

Rnaset2 $^{-/-}$Ifnar1 $^{-/-}$double-knockout mice. For the Rnaset ${ }^{-/-}$Ifnar $^{-/-}$ double-knockout mice, homozygous Rnaset $2^{-l-}$ mice were crossbred with homozygous B6.129S2-Ifnar tmlagt/Mmjax (Ifnar1 $^{-1-}$ ) kindly provided by Prof. Brück, Institute of Neuropathology, University Medical Center Göttingen ${ }^{59}$. Routine genotyping of the Ifnar $1^{-1-}$ knockout allele was performed by PCR with primer 1 (5'-CGAGGCGAAGTGGTTAAAAG-3'), primer 2 (5'-ACGGATCAAC CTCATTCCAC-3') and primer 3 (5'-AATTCGCCAATGACAAGACG-3') producing a product of $155 \mathrm{bp}$ for wild type Ifnarl allele and $250 \mathrm{bp}$ for the mutant allele.

Breeding and maintenance. Mice were bred and kept in the animal facility of the Max Planck Institute of Experimental Medicine (Göttingen, Germany) and in the facility of the University Medical Center Göttingen, Georg August University (Göttingen, Germany) with $21^{\circ} \mathrm{C}$ ambient temperature, 60-65\% humidity, a $12 \mathrm{~h}$ light/dark cycle and 2-5 mice per cage. All experiments were performed in accordance with the German animal protection law and with the permission of the Lower Saxony Federal State Office for Consumer Protection and Food Safety (LAVES) under the protocol No. 17-2697.

Protein extraction and western blotting. Heterozygous control and Rnaset2 ${ }^{-1-}$ mouse embryonic fibroblasts (MEFs) were prepared according to the protocol of Durkin et al. and maintained as primary cell cultures ${ }^{60}$. Briefly, day 13 embryos were harvested from the uterus of a female mouse, washed in PBS and the head and the red tissue (heart and liver) removed. The rest of the embryos were minced with scissor and scalpel and finally digested for up to $20 \mathrm{~min}$. at $37^{\circ} \mathrm{C}$ in $0.25 \%$ trypsinEDTA. The suspension was transferred to a $50 \mathrm{ml}$ tube and let sit for about $5 \mathrm{~min}$. Finally, the supernatant from three embryos was transferred to a T75 cell culture flask in MEF culture medium (Dulbecco's Modified Eagle's Medium (DMEM) containing $4.5 \mathrm{~g} / \mathrm{L}$ D-glucose (Life Technologies, Gibco ${ }^{\oplus}$ ) supplemented with $10 \%$ fetal bovine serum, $1 \times 200 \mathrm{mM}$ L-glutamine and $1 \mathrm{x}$ penicillin-streptomycin). The medium was exchange the next day. After a maximum of two passages, cells were harvested and lysed on ice in RIPA buffer containing Complete Protease and Phosphatase Inhibitor Cocktail (Roche). Protein concentration was determined by using the Interchim BC Assay Kit (Interchim) and bovine serum albumin was used as standard. Protein lysates were denatured by boiling in Tris-Glycine SDS sample buffer, separated by SDS-PAGE using 4-20\% Tris-Glycine Mini Gels (Thermo Fisher Scientific) and transferred onto $0.45 \mu \mathrm{m}$ nitrocellulose membranes (Merck). Subsequently, nitrocellulose membranes were blocked with $5 \%$ non-fat milk in TBS supplemented with $0.1 \%$ Tween 20 (TBST) for one hour at room temperature. Next, primary antibodies were applied in $5 \%$ non-fat milk in TBST overnight at $4^{\circ} \mathrm{C}$ (anti-RNaseT2 (1:500), Cloud-Clone Corp., Katy, USA; anti- $\beta$-actin (1:15.000), Sigma-Aldrich). On the next day, membranes were washed with TBST and each primary antibody was followed by incubation with either goat anti-rabbit or donkey anti-mouse secondary antibodies conjugated to HRP (Dianova), diluted 1:5000 in $5 \%$ non-fat milk in TBST for one hour at room temperature. Finally, chemiluminescent signal was revealed by using the Lumi-Light Western Blotting Substrate (Merck) and an ImageQuant LAS 4000 Mini imaging system (GE Healthcare). Equal loading of protein lysates was determined by $\beta$-actin Western blotting.

Histology and immunohistochemistry. For histology, immunohistochemistry (IHC) and organ weights, mixed-sex mice between the age of 4 and 10 months were anesthetized with ketamine $\left(250 \mathrm{mg} / \mathrm{kg}\right.$ i.p., Ursotamin ${ }^{\circledast}$ (Ketamin),
Serumwerk) and medetomidine ( $2 \mathrm{mg} / \mathrm{kg}$ i.p., Cepetor, CP-Pharma). Immediately after cardiac arrest, the animals were transcardially perfused with phosphatebuffered saline (PBS, pH 7.4) followed by $4 \%$ paraformaldehyde in PBS (pH 7.4). Organs were dissected out after perfusion and weighted on an analytical balance (2001 MP2, Sartorius). Organ specimens (liver, spleen, kidney, bone, and brain) were post-fixed in $4 \%$ paraformaldehyde in PBS for at least $72 \mathrm{~h}$ at $4{ }^{\circ} \mathrm{C}$, embedded in paraffin and cut in three to five $\mu \mathrm{m}$ sections.

First, detailed organ morphology was assessed by hematoxylin-eosin (HE) staining. For IHC, organ sections were de-paraffinized and steam-cooked in antigen target retrieval solution $\mathrm{pH} 9$ (Agilent Dako) according to the manufacturer's protocol. After washing the sections with Tris-buffered saline (TBS) supplemented with $0.025 \%$ Triton $^{\mathrm{TM}}$ X-100 (TBSTri), blocking was performed with $10 \%$ FCS with $1 \%$ BSA in TBS for $30 \mathrm{~min}$ at room temperature. Next, sections were incubated with primary antibodies: anti-CD41 (1:100, EPR4330, Abcam), rabbit anti-CD3 (1:150, mAb SP7, DCS), rat anti-Mac3 (1:200, mAb M3/84, BD Pharmingen) and anti-B220 (1:100, RA3-6B2, BD Pharmingen) in 1\% BSA in TBS overnight at $4{ }^{\circ} \mathrm{C}$. After washing the sections with TBSTri on the next day, endogenous peroxidase was inactivated by $0.3 \% \mathrm{H}_{2} \mathrm{O}_{2}$ incubation for $17 \mathrm{~min}$ at room temperature. Next, sections were incubated with horseradish peroxidase (HRP) conjugated goat anti-rabbit secondary antibody (1:300) (Dianova) for one hour at room temperature. The immunoreactivity was visualized using the SignalStain DAB Substrate Kit (Cell Signaling) by employing DAB as chromogen. Finally, sections were counterstained with Mayer's hemalum (Merck). Images were

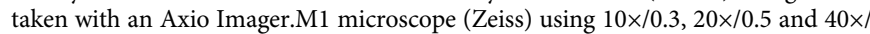
0.75 Plan-NEOFLUAR objectives and an Axiocam 105 color camera (Zeiss).

For the analysis of CNS tissue, histological staining comprised hematoxylineosin (HE), Luxol fast blue-periodic acid Schiff (LFB-PAS) and Bielschowsky silver impregnation. For immunohistochemistry, the following primary antibodies were used: mouse anti-glial fibrillary acidic protein (GFAP, 1:300, 173 011, Synaptic Systems), mouse anti-ionized calcium binding adapter molecule 1 (Ibal, 1:100, MABN92, Merck/Millipore), rabbit anti-NeuN (NeuN, 1:3000, ab177487, Abcam), rabbit anti-transmembrane protein 119 (TMEM119, 1:100, ab209064, Abcam), rabbit anti-interferon stimulating gene 15 (ISG15, 1:100, kindly provided by Marco Prinz), mouse anti-Platelet endothelial cell adhesion molecule (CD31, 1:10, DIA310M, Dianova), rat anti-myelin basic protein (MBP, 1:100, mAb12, Abcam), rabbit anti-CD3 (1:150, mAb SP7, DCS), rat anti-Mac3 (1:200, mAb M3/84, BD Pharmingen) and rat anti CD8a (1:100, 4SM15, Invitrogen). Antibody-binding was detected using a peroxidase/DAB protocol, as described above. For CD3-CD8 double immunohistochemistry, fluorescently labelled secondary antibodies were applied. Sections were evaluated using an Olympus BX51 light or combined light and fluorescent microscope. To analyze ISG15 expression on whole slide images of whole hemispheres color deconvolution (scikit-image, v0.18.1) of the DAB-ISG15 signal was performed (Supplementary Fig. 13) ${ }^{61}$.

Blood, urine, and bone marrow analysis. For the collection of blood samples mixed-sex mice between the age of 4 and 10 months were deeply terminal anesthetized with ketamine (250 mg/kg i.p.) and medetomidine ( $2 \mathrm{mg} / \mathrm{kg}$ i.p.) for cardiac puncture.

To obtain serum, whole blood samples were stored at room temperature for one to two hours before being separated by centrifugation and stored at $-20^{\circ} \mathrm{C}$. In order to measure clinical chemistry parameters, serum samples of 11 Rnaset $^{-1-}$ and 13-16 control mice were analyzed in the accredited interdisciplinary laboratory of the Institute of Clinical Chemistry of the University Medical Center Göttingen by standard protocols.

Peripheral blood counts were analyzed from ethylenediaminetetraacetic acid (EDTA)-anticoagulated whole blood samples in a threefold dilution using a Microsemi CRP Analyzer (Horiba) within four hours after blood withdrawal Knockout and control animals analyzed $n=13-20$, respectively.

For counting reticulocytes, EDTA-stabilized blood samples from each mouse were stained with brilliant cresyl blue (KABE REF017005) according to the manufacturer's recommendation, scratched out and analyzed by the "meander" 

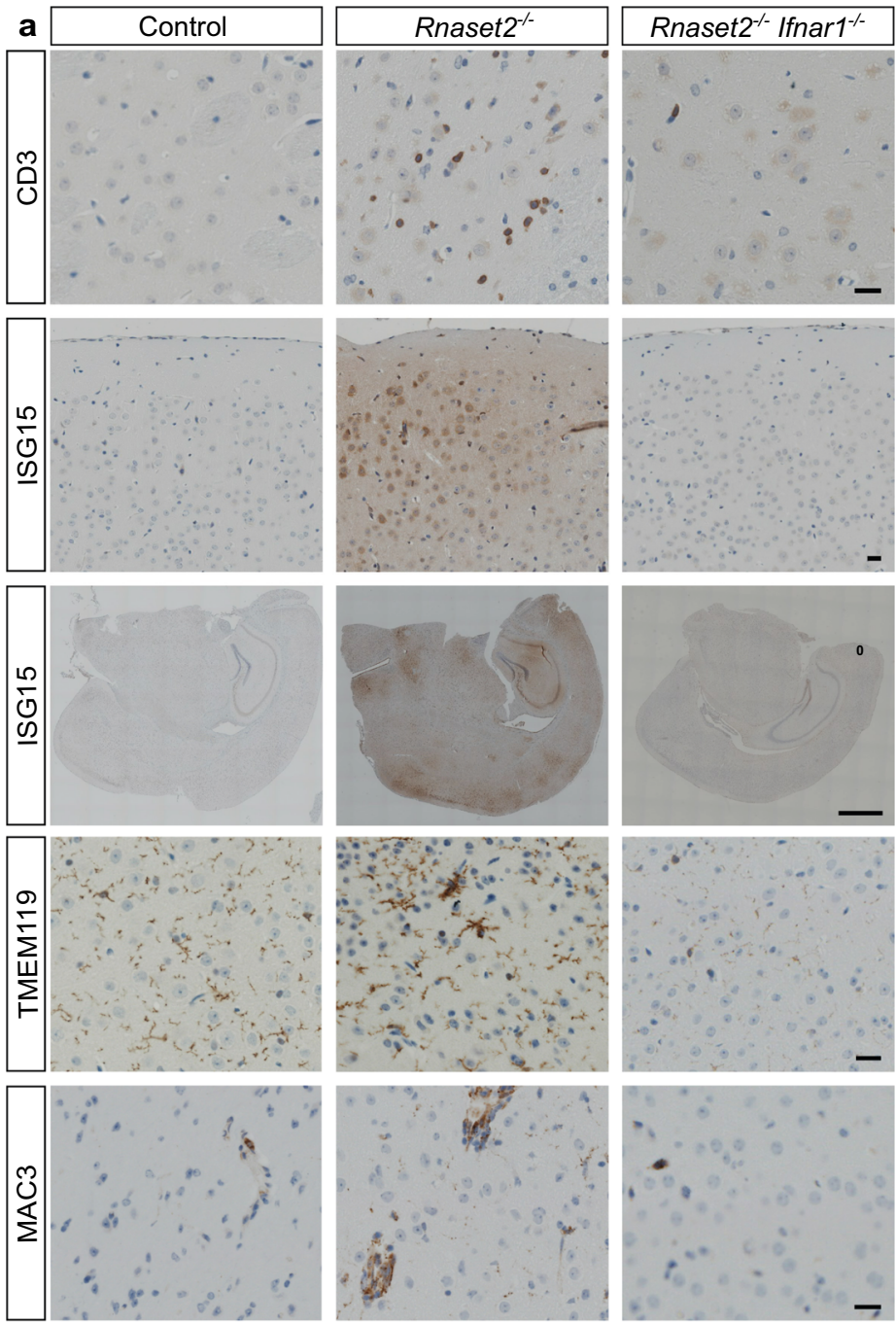

C $\mathrm{T}_{2}$ relaxation time in ex vivo MRI
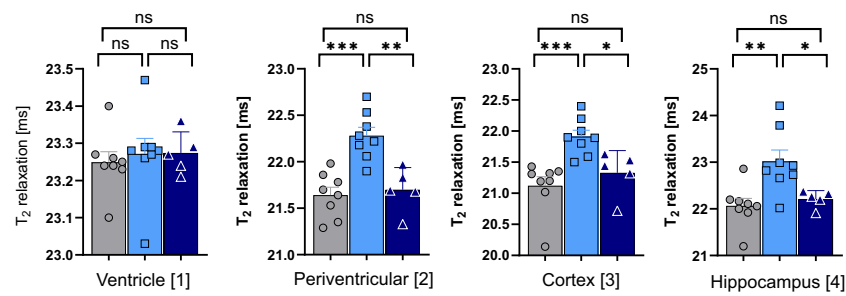

b

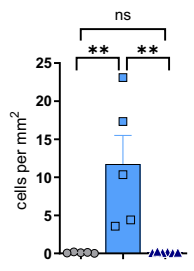

$\mathrm{CD}^{+}$cells
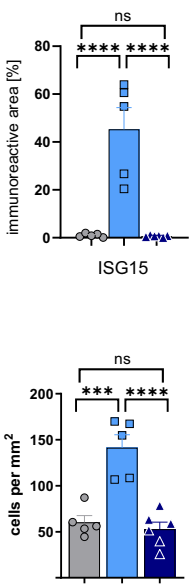

TMEM119+ cells

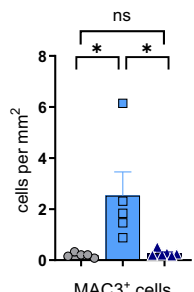

$\mathrm{MAC}^{+}$cells

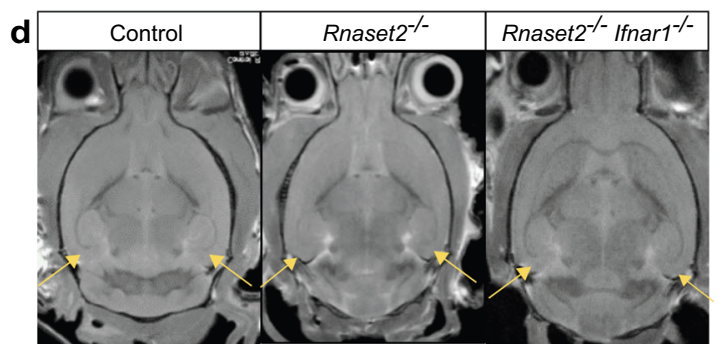

technique. At least 1000 erythrocytes were counted for each slide at a magnification of 1000 -fold to determine the number of reticulocytes.

Urine samples from Rnaset $2^{-I-}$ and control mice were collected during routine handling. In general proteins from urine samples are extracted by chloroformmethanol precipitation. Briefly, $20 \mu \mathrm{l}$ of urine was diluted with water to a final volume of $100 \mu$ l. Addition of 4 volumes methanol, 1 volume chloroform and 3 volumes water leads to extraction of total protein from the urine sample. After

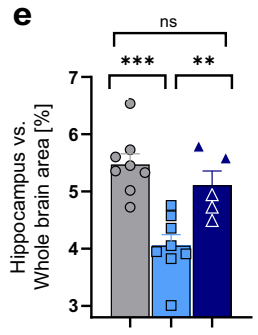

centrifugation, the supernatant was dried and proteins were denatured by boiling in Tris-Glycine SDS sample buffer and separated by SDS-PAGE using 4-20\% TrisGlycine Gel (NovexTM, Thermo Fisher Scientific). $300 \mathrm{ng}$ bovine serum albumin (BSA) was loaded as standard control and $10 \mu \mathrm{l}$ molecular marker (Pro Sieve Quad Proteinmarker 4.6-300 kDa, Biozym Scientific) was used.

The bone marrow from Rnaset $2^{-l-}$ and control mice was isolated after cardiac arrest from the femur. In brief, the femur was segregated from the mouse by 
Fig. 8 Ifnar $\mathbf{1}^{-/-}$knockout rescues Rnaset2 ${ }^{-/-}$related neuropathology. a Immunohistochemistry of rescued cerebral T cell infiltration (CD3), interferonstimulated gene 15 (ISG15) upregulation, microglial activation (TMEM119), and macrophages/activated microglia infiltration (MAC3) in Rnaset2-/Ifnar1 ${ }^{-/}$- mouse brain sections compared to control mouse and Rnaset2-/- mouse brain sections. Scale bar, $20 \mu \mathrm{M}$ and $1 \mathrm{~mm}$ for whole slices of ISG15. b Rescued cerebral inflammatory infiltration of $\mathrm{CD}^{+}$and $\mathrm{MAC}^{+}$cell densities determined in whole brain slices, $\mathrm{TMEM}^{+} 19^{+}$cell density of representative cortical regions, and \% of immunoreactive area for ISG15 in Rnaset2 ${ }^{-/-I f n a r 1^{-/}-(n=6) \text { compared to control }(n=5) \text { and Rnaset2 }}{ }^{-/-}(n=5)$. Images in a are representative for the 5-6 animals analyzed for each genotype. c $T_{2}$ relaxation time measurement in various brain regions of Rnaset2-/-Ifnar ${ }^{-/-}$ $(n=5)$, compared to control $(n=8)$ and Rnaset2 ${ }^{-/-}(n=8)$. For regions of interest (ROI) 1-4 selected for $\mathrm{T}_{2}$ relaxation time measurements see Fig. $5 \mathrm{~d}$. IFNAR1-deficiency resolved extension of $T_{2}$ relaxation in Rnaset2 ${ }^{-/}-$mice. Data of two independent experiments. $\mathbf{d}$ Representative magnetic resonance anatomical images in axial plane showing ventricular enlargement in Rnaset2 ${ }^{-/}$mice but not in Rnaset2 ${ }^{-/-I f n a r} 1^{-/-}$mice due to shrinkage of the hippocampus on both sides (arrows). e Percent area of hippocampus (left and right) with respect to whole brain area of $R$ naset2-/- $(n=8)$, control $(n=8)$ and Rnaset2 ${ }^{-/}$Ifnar1 ${ }^{-/}(n=6)$. Data depicted as the mean \pm SEM. $p$ values of one-way ANOVA are represented as ${ }^{\star \star \star \star} p<0.0001,{ }^{\star \star \star} p<0.001$, ${ }^{\star *} p<0.01,{ }^{*} p<0.05$ and not significant (ns) $p \geq 0.05$. MRI data from Rnaset2 ${ }^{-1-}$ and control mice are also depicted in Fig. 5c and d. Source data are provided as a Source data file.

cutting underneath the hip bone and above the knee. The tissue surrounding the femur was segregated, and the bone marrow was pushed out of the femur with the help of a bone marrow puncture needle. The bone marrow was placed on a microscopic slide, mixed up with $30 \mu \mathrm{l}$ physiological saline and scratched out. For detailed analysis, the bone marrow smears were stained with Giemsa stain in the accredited laboratory of the Institute of Pathology of the University Medical Center Göttingen by standard protocols.

FACS analysis and antibodies. Single-cell suspensions were prepared by mashing spleen mixed-sex mice between the age of 4 and 10 months through a $70 \mu \mathrm{m}$ cell strainer (Greiner Bio-One). Cells were washed with FACS buffer (PBS + 2\% FBS) and incubated for $15 \mathrm{~min}$ in Fc-blocking buffer and stained with the following antimouse antibodies (if not stated otherwise, all from BioLegend or eBioscience) for 30 min on ice protected from light: B220 (RA3-6B2), c-kit (2B8), CD34 (HM34), CD41 (MWReg30), CD44 (IM7), CD45 (30F11), CD71 (RI7217), CD105 (MJ7/18), CD150 (TC15-12F12.2), Gr1 (RB6-8C5), SCA1 (D7), Ter119 (TER-119), CD16/32 (93). 7-aminoactinomycin D (7-AAD, Thermo Fisher Scientific) was used to assess viability. Cells were washed twice and resuspended in FACS buffer, before flow cytometry analysis was performed using a BD LSRFortessa ${ }^{\mathrm{TM}}$ X-20 (BD Biosciences). Data were collected by BD FACSDiva Software v8.0.2 (BD Biosciences) and analyzed using the FlowJo ${ }^{\text {mot }}$ Software Version 10.6.1 (Becton, Dickinson and Company, 2019).

Hematopoietic fractions were determined according to the following immunophenotypes: Erythrocytes all B220 - and Ter119+: CD44- (R5), CD44+ low cellular volume (R4), CD44+ medium cellular volume (R3), CD44+ high cellular volume (R2). B220 - Ter119- Gr1- (lineage-) c-Kit+Sca1-(LKS-) CD34-CD16/32-, megakaryocyte-erythroid progenitor (MEP); LKS $-\mathrm{CD} 34+\mathrm{CD} 16 / 32-$, common myeloid progenitor (CMP); LKS $-\mathrm{CD} 34+\mathrm{CD} 16 /$ $32+$, granulocyte-macrophage progenitor (GMP); LKS $-\mathrm{CD} 150+\mathrm{CD} 41+$, megakaryocyte progenitor (MkP); LKS-CD41-CD16/32-CD150-CD105-, PreGM; LKS-CD41-CD16/32-CD150+CD105-, pre-megakaryocyte-erythroid (Pre-Meg-E); LKS-CD41-CD16/32-CD150+CD105+, pre-colony-forming unit erythroid (Pre-CFU-E); LKS-CD41-CD16/ 32-CD150-CD105+, CFUE.

Preparation of CNS mononuclear cells. Mixed-sex mice were transcardially perfused with $20 \mathrm{ml}$ PBS at the age of 4-10 months. Brain and spinal cord were removed and dissociated with the gentle MACS ${ }^{\mathrm{TM}}$ dissociator. The tissue was digested for $35 \mathrm{~min}$ with $2.5 \mathrm{mg} / \mathrm{ml}$ collagenase D (Roche) and $1 \mathrm{mg} / \mathrm{ml} \mathrm{DNaseI}$ (Roche) at $37^{\circ} \mathrm{C}$. Mononuclear cells were isolated by Percoll gradient centrifugation $(37 \% / 70 \%$, GE Healthcare) removed from the interphase, washed and subsequently blocked with $\alpha \mathrm{CD} 16 / 32$ (BioLegend, Clone 93 ) for $15 \mathrm{~min}$. The following antibodies were used (all from BioLegend or eBioscience): $\alpha \mathrm{CD} 3$ (145-2C11), aCD4 (RM4-5), $\alpha \mathrm{CD} 8$ (53-6.7), $\alpha \mathrm{CD} 11 \mathrm{~b}$ (M1/70), $\alpha \mathrm{CD} 19$ (1D3), $\alpha \mathrm{B} 220$ (RA36B2), $\alpha \mathrm{TCR} \beta$ (H57-597), ay $\delta$ TCR (GL3), $\alpha \mathrm{CD} 44$ (IM7), $\alpha \mathrm{CD} 62 \mathrm{~L}$ (MEL-14), aCD45 (30-F11), aLy6C (HK1.4), aLy6G (1A8), aCCR2 (R\&D 475301), aP2RY12 (S16007D), $\mathrm{aH} 2 \mathrm{~Kb} / \mathrm{Db}$ antibody (28-8-6), mouse IgG2a isotype control (MOPC$173)$. Flow cytometry analysis was performed using a BD LSRFortessa ${ }^{\mathrm{TM}} \mathrm{X}-20$ (BD Biosciences, CA) or a FACS Canto ${ }^{T M}$ II device (BD Biosciences, CA). Results were analyzed using FlowJo ${ }^{\mathrm{mt}}$ Software Version 10.6.1 (Becton, Dickinson and Company, 2019). Naïve CD8 T cells were defined as $\mathrm{CD} 45^{+} \mathrm{TCR} \beta^{+} \mathrm{CD} 8^{+} \mathrm{CD} 44^{\text {low }} \mathrm{CD} 62 \mathrm{~L}^{+}$, effector memory CD8 T cells as CD45 ${ }^{+} \mathrm{TCR} \beta^{+} \mathrm{CD} 8{ }^{+} \mathrm{CD} 44^{\mathrm{hi}} \mathrm{CD} 62 \mathrm{~L}^{-}$and central memory CD8 $\mathrm{T}$ cells as $\mathrm{CD} 45^{+} \mathrm{TCR} \beta^{+} \mathrm{CD} 8^{+} \mathrm{CD} 44^{\mathrm{hi}} \mathrm{CD} 62 \mathrm{~L}^{+}$. Inflammatory monocytes were defined as $\mathrm{CD} 45^{+} \mathrm{CD} 11 \mathrm{~b}^{+} \mathrm{Ly}_{6} \mathrm{C}^{\text {hi }} \mathrm{CCR} 2^{+}$cells. Microglia cells for MFI assessment were defined as $\mathrm{CD} 45^{\text {int }} \mathrm{CD}_{11 b^{+}} \mathrm{Ly}_{6} \mathrm{C}^{-} \mathrm{Ly}_{6 \mathrm{G}}{ }^{-}$ P2RY12 ${ }^{+}$.

\section{RNA extraction, reverse transcription, and quantitative real-time PCR}

(qPCR). A total RNA of six mixed-sex control and six Rnaset $2^{-1-}$ mice between 4 and 8 months were used for RNA extraction from the liver, spleen and kidney using FastGene RNA Premium Kit (Nippon Genetics) and from the brain using
peqGOLD TriFast ${ }^{\mathrm{TM}}$ (peqlab) according to the manufacturer's instructions. Organs were isolated from Rnaset $2^{-1-}$ and control mice after perfusion with PBS (pH 7.5). RNA quality was verified by gel electrophoresis and optical density (OD) measurement. For first-strand cDNA synthesis, $2 \mu \mathrm{g}$ of RNA was reverse transcribed using SuperScript III First-Strand Synthesis System (Invitrogen) according to the manufacturer's recommendation.

Quantitative PCR for Ifi27, Ifi44, Ifit1, Isg15, Rsad2, Siglec1, Usp18, Cxcl10, Irf7, Ifih $1, D d x 58$ and Rplp0 and Tbp (as internal controls) were performed using PrimeTime qPCR Assays listed in Supplementary Table 2 and ordered by Integrated DNA Technology (IDT). All reactions were done in triplicates on a QuantStudio 3 Real-Time PCR System (Applied Biosystems) according to the manufacturer's recommendations in reactions containing $20 \mathrm{ng}$ cDNA. The relative gene expression values were quantified according to the $\Delta \Delta_{\mathrm{CT}}$ method $^{62}$ by QuantStudio ${ }^{\text {nix }}$ Design \& Analysis Software v1.4.3, and the values of controls were normalized to 1 .

Detection of anti-nuclear antibodies. The standard method for detecting antinuclear antibodies (ANA) is the indirect immunofluorescence test (IIFT). ANA measurements were performed by EUROIMMUN AG (Lübeck, Germany) using the HEp-2 IIFT Dog (IgG) test kit and an anti-mouse-IgG-FITC antibody. Rnaset $2^{-I-}$ and control mouse sera were tested. In brief, BIOCHIPS coated with HEp2 cells were incubated with differently diluted mouse sera. In a positive reaction, specific antibodies of the IgG class bind to antigens and in a second step, attached antibodies are stained with fluorescein-labelled anti-mouse antibodies and visualized using a fluorescence microscope. Results are reported as titres and titres are expressed as ratios obtained by dilution of the sample with saline. To evaluate antinuclear antibody prevalence, samples were further titrated until a fluorescence signal was just visible.

Ex vivo magnetic resonance imaging. For cerebral magnetic resonance imaging (MRI), mixed-sex mice between the age of 4 and 7 month were anesthetized with ketamine $(250 \mathrm{mg} / \mathrm{kg}$ i.p.) and medetomidine $(2 \mathrm{mg} / \mathrm{kg}$ i.p.). Immediately after cardiac arrest, the animals were transcardially perfused with PBS ( $\mathrm{pH} 7.4)$ followed by $4 \%$ paraformaldehyde in PBS (pH 7.4). Mice heads were separated and postfixed in $4 \%$ paraformaldehyde in PBS (pH 7.4) for at least one week at $4{ }^{\circ} \mathrm{C}$. Before MRI measurements, the head were embedded in Fomblin (perfluoropolyether). All MRI measurements were conducted on a $300 \mathrm{MHz}(7 \mathrm{~T})$ vertical wide bore system, using a transmit/receive birdcage radiofrequency coil with an inner diameter of $25 \mathrm{~mm}$ and a $1 \mathrm{Tm}^{-1}$ gradient insert (Bruker). The system was interfaced to a Linux operating system running Topspin 2.0 and Para Vision 3.0 imaging software (Bruker Biospin). Before each measurement, the magnetic field homogeneity was optimized by shimming. Each session of measurements started with a multi-slice orthogonal gradient-echo sequence for position determination and selection of the desired region for subsequent experiments. $T_{2}$-weighted $M R$ images were acquired by a rapid acquisition with relaxation enhancement (RARE) sequence, which employs a single excitation step followed by the collection of multiple phaseencoded echoes ${ }^{63}$. Basic measurement parameters used for the RARE sequence were as follows: effective echo time $=18.1 \mathrm{~ms}$; repetition time $=5 \mathrm{~s}$; flip angle $=$ $90^{\circ}$; averages $=32$ (for sagittal and axial) or 64 (for coronal); and RARE factor (echo train length) $=4$; image slice thickness $=0.5 \mathrm{~mm}$. The field of view was $2.5 \times 2.5 \mathrm{~cm}^{2}$, with an image matrix of $256 \times 256$. This yields an effective in-plane resolution of $\sim 97 \mu \mathrm{m}$. For measurement of brain areas, the desired region of interest was drawn on the image and areas were computed using an image sequence analysis (ISA) tool package (Paravision 5, Bruker). The data were exported to OriginPro v. 8 (OriginLab) for further analysis and percentage of specific area with respect to whole brain area was calculated. One-way ANOVA (Tukey's post-test) for comparison of mean between groups was performed. Levene's test was performed for homogeneity of variance analysis.

For transverse relaxation time $\left(\mathrm{T}_{2}\right)$ measurement, a standard multi-slice multiecho (MSME) sequence was used. This sequence is based on the Carr-Purcell Meiboom-Gill (CPMG) sequence, where transverse magnetization of a $90^{\circ}$ pulse is 
refocused by a train of $180^{\circ}$ pulses generating a series of echoes. The following imaging parameters were used: nominal flip angles $=90^{\circ}$ and $180^{\circ}$, and a train of 12 echoes with TEs ranging from $8.17 \mathrm{~ms}$ to $98 \mathrm{~ms}$ with $8.17 \mathrm{~ms}$ echo-spacing, $\mathrm{TR}=2 \mathrm{~s}$, slice thickness $=0.5 \mathrm{~mm}$; number of slices 8 and a matrix size $256 \times 256$ pixels. For calculation of $\mathrm{T}_{2}$ relaxation time, regions of interest (ROIs) were drawn at desired locations within the brain using an image sequence analysis (ISA) tool package (Paravision 5, Bruker). Monoexponential fitting was then used to calculate $\mathrm{T}_{2}$ using a monoexponential fit function $\left[\mathrm{y}=\mathrm{A}+\mathrm{C}^{*} \exp \left(-\mathrm{t} / \mathrm{T}_{2}\right)\right]$, where $\mathrm{A}=$ Absolute bias, $\mathrm{C}=$ signal intensity, $\mathrm{T}_{2}=$ transverse relaxation time. Means and standard deviation for $\mathrm{T}_{2}$ relaxation times for each ROI were calculated.

In vivo magnetic resonance imaging. For this analysis two male Rnaset $2^{-/-}$and two male control mice at age of 10 months were assessed. Mice were anesthetized with ketamine $(60 \mathrm{mg} / \mathrm{kg}$, i.p.) and medetomidine $(0.4 \mathrm{mg} / \mathrm{Kg}$, i.p.), subsequently intubated and maintained under anesthesia by active ventilation with isoflurane $(0.5-1.75 \%$ in ambient air and oxygen (1:1)). MRI data were acquired at a magnetic field strength of 9.4 Tesla (94/30 BioSpec, Bruker BioSpin) using a saddle shaped 4-channel phased array coil for signal detections. Multi-slice T2-weighted images* (RARE, TR/effective TE $=6843 / 55 \mathrm{~ms}$, number of echoes 8 ) were obtained with a special resolution of $40 \times 40 \times 300 \mu^{3}$. For dynamic contrast enhanced MRI $(\mathrm{DCM})$ a 3D T1-weighted data set (FLASH, TR/TE $=18 / 3.4$, flip angle $=8^{\circ}$, $100 \mu \mathrm{m}$ isotropic spatial resolution) was acquired before $\left(\mathrm{S}_{0}\right)$ and $20 \mathrm{~min}$ after $\left(\mathrm{S}_{\mathrm{C}}\right)$ intravenous injection (via tail vein catheter) of $0.3 \mathrm{mmol} / \mathrm{Kg}$ body weight $\mathrm{Gd}$ DTPA (Magnevist ${ }^{\oplus}$, Bayer Vital GmbH, diluted to $0.1 \mathrm{mM}$ in physiological saline). Signal enhancement in percentage was calculated as followed: $\left(\mathrm{S}_{\mathrm{C}}-\mathrm{S}_{0}\right) / \mathrm{S}_{0} * 100$. 3D-reconstruction of the brain and the lateral ventricles was performed based on magnetisation transfer-weighted data sets (3D FLASH, TR/TE $=15.2 / 3.4 \mathrm{~ms}$, flip angle $=5^{\circ}$, Gaussian pulse with an off-resonance frequency of $3000 \mathrm{~Hz}$ and a power of $6 \mu \mathrm{T}, 100 \mu \mathrm{m}$ isotropic spatial resolution) using the software package Amira 6.2.0 (Thermo Fisher Scientific) for semiautomatic segmentation.

Behavior testing. For all behavioral analysis a cohort of 17 Rnaset $^{-1-}$ and 15 control mice, at an age of 4 months, with mixed sex, was assessed.

The balance beam task was used to assess balance and general motor performance. A wooden beam of $1 \mathrm{~cm}$ diameter and a length of $50 \mathrm{~cm}$ was attached to two $9 \times 15 \mathrm{~cm}$ support columns, which were elevated at a height of $44 \mathrm{~cm}$ above a padded surface. Mice were positioned on the beam in a central position and released. During a single day of testing, each mouse was given three consecutive $60 \mathrm{~s}$ trials with at least $10 \mathrm{~min}$ intervals in between. For mice remaining on the beam for the entire trial period or managing to reach one of the escape platforms, the maximum time of $60 \mathrm{~s}$ was recorded and an average latency to fall was calculated ${ }^{64}$.

Neuromuscular abilities and muscle strength were tested using the inverted grid test. The testing apparatus consisted of a $45 \mathrm{~cm} \times 30 \mathrm{~cm}$ wire grid $45 \mathrm{~cm}$ wire grid with $1 \mathrm{~mm}$ wires and a grid spacing of $1 \mathrm{~cm}$. The grid was suspended $40 \mathrm{~cm}$ above a padded surface. Latency to fall was recorded during a single $60 \mathrm{~s}$ trial after a mouse had been placed onto the center of the grid and turned upside down. If the mice remained on the grid for the entire trial, the maximum time of $60 \mathrm{~s}$ was recorded ${ }^{65}$.

The novel object recognition (NOR) test relies on three independent sessions: one habituation session (Day0), one training session (Day1), and one test session (Day2), 5 min each, performed on 3 consecutive days. The open field test was used as habituation phase, thereby assessing both exploratory behavior and locomotor activity. Mice were tested using an open field box made of grey plastic with $50 \mathrm{~cm} \times 50 \mathrm{~cm}$ surface area and $38 \mathrm{~cm}$-high walls. Monitoring was accomplished by an automated tracking system (ANY-maze v5.1, Stoelting Co.) and the running speed was recorded during 5 -min sessions. The training session for the NOR analysis simply involves visual exploration of two identical objects, while one of these objects is replaced with a novel object in the test session. Because rodents have an innate preference for novelty, a rodent that remembers the familiar object will spend more time exploring the novel object. On day 1 , two similar objects were placed in different quadrants of the open field arena, and the mouse could move freely for $5 \mathrm{~min}$. In order to diminish odor cues, the arena and objects were cleaned with $70 \%$ ethanol solution after each test session. On testing day, one of the two objects from training day was replaced by a novel object. Mice were again allowed to explore freely for a period of $5 \mathrm{~min}$. For data analysis, animals with exploration times below $5 \mathrm{~s}$ in one period of $5 \mathrm{~min}$ were excluded. The recognition performances were quantified using the Discrimination Ratio (DR), measured as the differences between novel $\left(\mathrm{T}_{\text {novel }}\right)$ and familiar $\left(\mathrm{T}_{\text {familiar }}\right)$ object exploration times in proportion to the animal's total exploration time $\left(\mathrm{T}_{\text {total }}\right):\left(\mathrm{T}_{\text {novel }}-\mathrm{T}_{\text {familiar }}\right) /$ $\mathrm{T}_{\text {total. }}$. A DR equivalent to 0 indicates an equal exploration of the novel and familiar objects, while a positive or a negative value points to a preferential exploration of the novel and familiar object ${ }^{66}$.

Spontaneous alternation rates were assessed using a cross maze built from grey plastic material which had four arms arranged in a $90^{\circ}$ position. Each arm was $30 \mathrm{~cm}$ in length, with $8 \mathrm{~cm}$ width and a height of $15 \mathrm{~cm}$. During 10 min test sessions, each mouse was randomly placed in one arm and allowed to move freely through the maze. Alternation was defined as successive entries into the four arms in overlapping quadruple sets (e.g., 1, 3, 2, 4 or 2, 3, 4, 1 but not 1, 2, 3, 1). Percent of spontaneous alternation was defined as the ratio of actual (=total alternations) to possible (=total arm entries -3$)$ number of alternations $\times 100$. In order to diminish odor cues, the maze was cleaned with $70 \%$ ethanol solution ${ }^{67}$.
Single nuclei RNA sequencing analysis. For RNA sequencing analysis two mixed-sex Rnaset $2^{-I-}$ and control mice at the age of 8 months were assessed. Mice were deeply anesthetized with ketamine and medetomidine and transcardially perfused with phosphate-buffered saline (PBS, $\mathrm{pH}$ 7.4). After cervical dislocation, the whole brain was removed from the skull and the preparation of the hippocampus and caudate putamen was performed in a petri dish filled with ice cold PBS buffer under a binocular. Tissue sample from two hemispheres of different mice with the same genotype were pooled and immediately frozen on liquid nitrogen and stored at $-80^{\circ} \mathrm{C}$ until further processing. Nuclei from frozen mouse brain tissues were isolated according to previously published protocol with modifications ${ }^{68}$. Briefly, froze tissues were Dounce homogenized in $500 \mu \mathrm{lEZ}$ prep lysis buffer (Sigma NUC101) supplemented with 1:200 RNAse inhibitor (Promega, $\mathrm{N} 2615$ ) for 45 times in a $1.5 \mathrm{~mL}$ Eppendorf tube using micro pestles. The volume was increased with lysis buffer up to $2000 \mu \mathrm{l}$ and incubated on ice for $5 \mathrm{~min}$. Lysates were centrifuged for $5 \mathrm{~min}$ at $500 \times g$ at $4{ }^{\circ} \mathrm{C}$ and supernatants were discarded. The pellet was resuspended into $2000 \mu \mathrm{l}$ lysis buffer and incubated for $5 \mathrm{~min}$ on ice. After 5 min centrifugation $\left(500 \times g\right.$ at $\left.4{ }^{\circ} \mathrm{C}\right)$, the resulting pellet was resuspended into $1500 \mu \mathrm{l}$ nuclei suspension buffer (NSB, $0.5 \%$ BSA, 1:100 Roche protease inhibitor, 1:200 RNAse inhibitor in $1 \times \mathrm{PBS}$ ) and centrifuged again for $5 \mathrm{~min}$ $\left(500 \times g\right.$ at $\left.4{ }^{\circ} \mathrm{C}\right)$. The pellet was finally resuspended into $500 \mu \mathrm{l}$ NSB and stained with 7AAD (Invitrogen, Cat: 00-6993-50). Single nuclei were sorted using BD FACS Aria III sorter. Sorted nuclei were counted in Countess II FL Automated Counter. 5000 single nuclei per sample were used for GEM generation, barcoding, and cDNA libraries according to $10 \times$ Chromium Next GEM Single Cell 3 ' Reagent v3.1 protocol. Pooled libraries were sequenced in Illumina NextSeq 550 in order to achieve $>50,000$ reads/nuclei.

Gene counts were obtained by aligning reads to the $\mathrm{mm} 10$ genome (NCBI:GCA 000001635.8) (GRCm38.p6) using CellRanger software v.4.0.0 (10XGenomics). The CellRanger count pipeline was used to generate a gene-count matrix by mapping reads to the pre-mRNA as reference to account for unspliced nuclear transcripts.

The SCANPY package v1.7.2 was used for pre-filtering, normalization and clustering ${ }^{68}$. Initially, cells that reflected low-quality cells (either too many or too few reads, cells isolated almost exclusively, cells expressing less than $10 \%$ of housekeeping genes) were excluded ${ }^{69}$. Next, counts were scaled by the total library size multiplied by 10.000 and transformed to log space. Highly variable genes were identified based on dispersion and mean, the technical influence of the total number of counts was regressed out, and the values were rescaled. Differential expression analysis was performed using the rank_genes_groups function as implemented in SCANPY.

Principal component analysis (PCA) was performed on the variable genes, and UMAP was run on the top 50 principal components $(\mathrm{PCs})^{70}$. The top 50 PCs were used to build a $\mathrm{k}$-nearest-neighbours cell-cell graph with $\mathrm{k}=100$ neighbours. Subsequently, spectral decomposition over the graph was performed with 50 components, and the Leiden graph-clustering algorithm was applied to identify cell clusters. We confirmed that the number of PCs captures almost all the variance of the data. For each cluster, we assigned a cell-type label using manual evaluation of gene expression for sets of known marker genes. Violin plots for marker genes were created using the stacked_violin function as implemented in SCANPY. GO-term analysis was performed using ShinyGOVo. $60^{71}$. Data files of all differentially expressed genes and GO-term analysis are accessible at Supplementary Data 1.

Statistics and reproducibility. Statistical analysis was performed with GraphPad Prism version 8.3.0 (GraphPad Software Inc, San Diego, CA) using two-tailed Student's t-test, two-tailed Welsh's test, one-way ANOVA and two-way ANOVA followed by Tukey's multiple comparison test unless indicated otherwise. For all tests, $p$-values less than 0.05 were considered statistically significant. Asterisks indicate the magnitude of the statistical significance: ${ }^{*} p<0.05,{ }^{*} p<0.01$, ${ }^{* * *} p<0.001,{ }^{* * * *} p<0.0001$. ns (not significant) $p \geq 0.05$. Error bars indicate the mean with standard error of the mean (SEM).

Survival curves are computed using the open source python package lifelines (version 0.26.0, doi:10.5281/zenodo.4816284) and confidence intervals are estimated using the implemented exponential Greenwood method. The alpha level was corrected using Bonferroni's method by the number of comparisons $($ alpha $=0.05 / 3)$.

Immunofluorescence recordings were partially adjusted in brightness and contrast to different degrees, rotated and cropped using the OMERO.figure add-on (https://www.openmicroscopy.org/omero/figure/). Especially in Fig. 7f GFAP, Iba-1 and CD31 were adjusted for optimal morphology visualization while ISG15 was recorded and displayed using exactly the same conditions for control and Rnaset $2^{-1-}$

Reporting summary. Further information on research design is available in the Nature Research Reporting Summary linked to this article.

\section{Data availability}

Raw data for all results/figures presented in this article, including Supplementary Figures, are available in the Source data file. Any additional data are available from the corresponding author upon reasonable request. Raw single nuclei RNA sequencing data are accessible from the NCBI GEO database using the accession number GSE180138. 
Detailed annotation tables for these datasets are provided in Supplementary Data 1. Mus musculus reference genome (GRCm38.p6) use for single RNA nuclei study is accessible from NCBI GenBank under the accession numbers GCA_000001635.26. Reference sequence for Rnaset $2 a$ and Rnaset $2 b$ gene are accessible from NCBI GenBank under the accession numbers NM_001083938.3 and NM_026611.2, respectively. Source data are provided with this paper.

Received: 24 May 2020; Accepted: 14 October 2021; Published online: 11 November 2021

\section{References}

1. Henneke, M. et al. RNASET2-deficient cystic leukoencephalopathy resembles congenital cytomegalovirus brain infection. Nat. Genet. 41, 773-775 (2009).

2. Henneke, M. et al. Cystic leukoencephalopathy without megalencephaly: a distinct disease entity in 15 children. A distinct disease entity in 15 children. Neurology 64, 1411-1416 (2005)

3. Tonduti, D. et al. Clinical, radiological and possible pathological overlap of cystic leukoencephalopathy without megalencephaly and Aicardi-Goutières syndrome. Eur. J. Paediatr. Neurol. 20, 604-610 (2016).

4. Hofer, M. J. \& Campbell, I. L. Immunoinflammatory diseases of the central nervous system-the tale of two cytokines. Br. J. Pharmacol. 173, 716-728 (2016).

5. Dussaix, E., Lebon, P., Ponsot, G., Huault, G. \& Tardieu, M. Intrathecal synthesis of different alpha-interferons in patients with various neurological diseases. Acta Neurol. Scand. 71, 504-509 (1985).

6. Curtsinger, J. M., Lins, D. C., Johnson, C. M. \& Mescher, M. F. Signal 3 tolerant CD8 T cells degranulate in response to antigen but lack granzyme B to mediate cytolysis. J. Immunol. 175, 4392-4399 (2005).

7. Kolumam, G. A., Thomas, S., Thompson, L. J., Sprent, J. \& Murali-Krishna, K. Type I interferons act directly on CD8 T cells to allow clonal expansion and memory formation in response to viral infection. J. Exp. Med. 202, 637-650 (2005).

8. Akwa, Y. et al. Transgenic expression of IFN-alpha in the central nervous system of mice protects against lethal neurotropic viral infection but induces inflammation and neurodegeneration. J. Immunol. 161, 5016-5026 (1998).

9. Campbell, I. L. et al. Structural and functional neuropathology in transgenic mice with CNS expression of IFN- $\alpha 1$ Published on the World Wide Web on 17 March 1999.1. Brain Res. 835, 46-61 (1999).

10. Bartok, E. \& Hartmann, G. Immune sensing mechanisms that discriminate self from altered self and foreign nucleic acids. Immunity 53, 54-77 (2020).

11. Tang, D., Kang, R., Coyne, C. B., Zeh, H. J. \& Lotze, M. T. PAMPs and DAMPs: signal 0s that spur autophagy and immunity. Immunological Rev. 249, 158-175 (2012).

12. Rodero, M. P. \& Crow, Y. J. Type I interferon-mediated monogenic autoinflammation: the type I interferonopathies, a conceptual overview. J. Exp. Med. 213, 2527-2538 (2016).

13. Crow, Y. J. et al. Mutations in the gene encoding the 3 ' -5 ' DNA exonuclease TREX1 cause Aicardi-Goutières syndrome at the AGS1 locus. Nat. Genet. 38, 917-920 (2006).

14. Rice, G. I. et al. Gain-of-function mutations in IFIH1 cause a spectrum of human disease phenotypes associated with upregulated type I interferon signaling. Nat. Genet. 46, 503-509 (2014).

15. Livingston, J. H. \& Crow, Y. J. Neurologic phenotypes associated with mutations in TREX1, RNASEH2A, RNASEH2B, RNASEH2C, SAMHD1, ADAR1, and IFIH1: aicardi-goutières syndrome and beyond. aicardi-goutieres syndrome and beyond. Neuropediatrics 47, 355-360 (2016).

16. Schlee, M. \& Hartmann, G. Discriminating self from non-self in nucleic acid sensing. Nat. Rev. Immunol. 16, 566-580 (2016).

17. Haud, N. et al. rnaset2 mutant zebrafish model familial cystic leukoencephalopathy and reveal a role for RNase T2 in degrading ribosomal RNA. Proc. Natl Acad. Sci. USA 108, 1099-1103 (2011).

18. Hamilton, N. et al. The failure of microglia to digest developmental apoptotic cells contributes to the pathology of RNASET2-deficient leukoencephalopathy. Glia 68, 1531-1545 (2020)

19. Huang, J., Liu, P. \& Wang, G. Regulation of mitochondrion-associated cytosolic ribosomes by mammalian mitochondrial ribonuclease T2 (RNASET2). J. Biol. Chem. 293, 19633-19644 (2018).

20. Irie, M. Structure-function relationships of acid ribonucleases: lysosomal, vacuolar, and periplasmic enzymes. Pharmacol. Ther. 81, 77-89 (1999).

21. Greulich, W. et al. TLR8 is a sensor of RNase T2 degradation products. Cell 179, 1264-1275.e13 (2019).

22. Ostendorf, T. et al. Immune sensing of synthetic, bacterial, and protozoan RNA by toll-like receptor 8 requires coordinated processing by RNase T2 and RNase 2. Immunity 52, 591-605.e6 (2020).
23. Goldmann, T. et al. USP18 lack in microglia causes destructive interferonopathy of the mouse brain. EMBO J. 34, 1612-1629 (2015).

24. Hartner, J. C., Walkley, C. R., Lu, J. \& Orkin, S. H. ADAR1 is essential for the maintenance of hematopoiesis and suppression of interferon signaling. Nat. Immunol. 10, 109-115 (2009).

25. Funabiki, M. et al. Autoimmune disorders associated with gain of function of the intracellular sensor MDA5. Immunity 40, 199-212 (2014).

26. Rego, S. L. et al. TREX1 D18N mice fail to process erythroblast DNA resulting in inflammation and dysfunctional erythropoiesis. Autoimmunity 51, 333-344 (2018).

27. Morita, M. et al. Gene-targeted mice lacking the Trex1 (DNase III) 3'-5' DNA exonuclease develop inflammatory myocarditis. Mol. Cell. Biol. 24, 6719-6727 (2004).

28. Rehwinkel, J. et al. SAMHD1-dependent retroviral control and escape in mice. EMBO J. 32, 2454-2462 (2013).

29. Hiller, B. et al. Mammalian RNase $\mathrm{H} 2$ removes ribonucleotides from DNA to maintain genome integrity. J. Exp. Med. 209, 1419-1426 (2012).

30. Cox, A. et al. A new standard genetic map for the laboratory mouse. Genetics 182, 1335-1344 (2009).

31. van Eyck, L. et al. Brief report: IFIH1 Mutation causes systemic lupus erythematosus with selective IgA deficiency. Arthritis Rheumatol. 67, 1592-1597 (2015)

32. Dale, R. C., Tang, S. P., Heckmatt, J. Z. \& Tatnall, F. M. Familial systemic lupus erythematosus and congenital infection-like syndrome. Neuropediatrics 31, 155-158 (2000)

33. Roy, U. et al. Degeneration of the suprachiasmatic nucleus in an Alzheimer's disease mouse model monitored by in vivo magnetic resonance relaxation measurements and immunohistochemistry. J. Alzheimer's Dis. 69, 363-375 (2019).

34. Aw, E., Zhang, Y. \& Carroll, M. Microglial responses to peripheral type 1 interferon. J. Neuroinflammation 17, 340 (2020).

35. Wang, W., Zhao, F., Ma, X., Perry, G. \& Zhu, X. Mitochondria dysfunction in the pathogenesis of Alzheimer's disease: recent advances. Mol. Neurodegener. 15, $30(2020)$

36. Lezi, E. \& Swerdlow, R. H. Mitochondria in neurodegeneration. Adv. Exp. Med. Biol. 942, 269-286 (2012).

37. Lin, M. T. \& Beal, M. F. Mitochondrial dysfunction and oxidative stress in neurodegenerative diseases. Nature 443, 787-795 (2006).

38. Miyashita, M., Oshiumi, H., Matsumoto, M. \& Seya, T. DDX60, a DEXD/H box helicase, is a novel antiviral factor promoting RIG-I-like receptormediated signaling. Mol. Cell. Biol. 31, 3802-3819 (2011).

39. Falcão, A. M. et al. Disease-specific oligodendrocyte lineage cells arise in multiple sclerosis. Nat. Med. 24, 1837-1844 (2018).

40. Ward, L. A. \& Massa, P. T. Neuron-specific regulation of major histocompatibility complex class I, interferon- $\beta$, and anti-viral state genes. J. Neuroimmunol. 58, 145-155 (1995).

41. Sinkevicius, K. W. et al. RNaseT2 knockout rats exhibit hippocampal neuropathology and deficits in memory. Dis. models mechanisms 11, 1-12 (2018).

42. Pardieck, I. N., Beyrend, G., Redeker, A. \& Arens, R. Cytomegalovirus infection and progressive differentiation of effector-memory T cells. F1000Res. 7, 1554 (2018).

43. van der Most, R. G., Murali-Krishna, K. \& Ahmed, R. Prolonged presence of effector-memory CD8 T cells in the central nervous system after dengue virus encephalitis. Int. Immunol. 15, 119-125 (2003).

44. Xiao, N. et al. cGAS activation causes lupus-like autoimmune disorders in a TREX1 mutant mouse model. J. Autoimmun. 100, 84-94 (2019).

45. Gate, D. et al. Clonally expanded CD8 T cells patrol the cerebrospinal fluid in Alzheimer's disease. Nature 577, 399-404 (2020).

46. Ritzel, R. M. et al. Age-associated resident memory CD8 T cells in the central nervous system are primed to potentiate inflammation after ischemic brain injury. J. Immunol. 196, 3318-3330 (2016).

47. Raivich, G. et al. Immune surveillance in the injured nervous system: T-lymphocytes invade the axotomized mouse facial motor nucleus and aggregate around sites of neuronal degeneration. J. Neurosci. 18, 5804-5816 (1998).

48. Ip, C. W. et al. Immune cells contribute to myelin degeneration and axonopathic changes in mice overexpressing proteolipid protein in oligodendrocytes. J. Neurosci. 26, 8206-8216 (2006).

49. Yoshida, H., Okabe, Y., Kawane, K., Fukuyama, H. \& Nagata, S. Lethal anemia caused by interferon-beta produced in mouse embryos carrying undigested DNA. Nat. Immunol. 6, 49-56 (2005).

50. Hartner, J. C. et al. Liver disintegration in the mouse embryo caused by deficiency in the RNA-editing enzyme ADAR1. J. Biol. Chem. 279, 4894-4902 (2004).

51. Ben-Shoshan, S. O. et al. ADAR1 deletion induces NFkB and interferon signaling dependent liver inflammation and fibrosis. RNA Biol. 14, 587-602 (2017). 
52. Liddicoat, B. J. et al. Adenosine-to-inosine RNA editing by ADAR1 is essential for normal murine erythropoiesis. Exp. Hematol. 44, 947-963 (2016).

53. Rodero, M. P. et al. Type I interferon-mediated autoinflammation due to DNase II deficiency. Nat. Commun. 8, 2176 (2017).

54. Chan, M. P. et al. DNase II-dependent DNA digestion is required for DNA sensing by TLR9. Nat. Commun. 6, 5853 (2015).

55. Pawaria, S. et al. Cutting edge: DNase II deficiency prevents activation of autoreactive B cells by double-stranded DNA endogenous ligands. J. Immunol. 194, 1403-1407 (2015).

56. Jakobs, C., Perner, S. \& Hornung, V. AIM2 Drives joint inflammation in a Self-DNA triggered model of chronic polyarthritis. PLOS ONE 10, e0131702 (2015).

57. Schwefel, K. et al. Biallelic CCM3 mutations cause a clonogenic survival advantage and endothelial cell stiffening. J. Cell. Mol. Med. 23, 1771-1783 (2019).

58. Sung, Y. H. et al. Highly efficient gene knockout in mice and zebrafish with RNA-guided endonucleases. Genome Res. 24, 125-131 (2014).

59. Müller, U. et al. Functional role of type I and type II interferons in antiviral defense. Science 264, 1918-1921 (1994).

60. Durkin, M. E., Qian, X., Popescu, N. C. \& Lowy, D. R. Isolation of mouse embryo fibroblasts. Bio-Protoc. 3, 1-5 (2013)

61. Ruifrok, A. C., Katz, R. L. \& Johnston, D. A. Comparison of quantification of histochemical staining by hue-saturation-intensity (HSI) transformation and color-deconvolution. Appl. Immunohistochem. Mol. Morphol. 11, 85-91 (2003).

62. Livak, K. J. \& Schmittgen, T. D. Analysis of relative gene expression data using real-time quantitative PCR and the 2(-Delta Delta C(T)) Method. Methods 25 , 402-408 (2001).

63. Hennig, J., Nauerth, A. \& Friedburg, H. RARE imaging: a fast imaging method for clinical MR. Magn. Reson. Med. 3, 823-833 (1986).

64. Hüttenrauch, M., Walter, S., Kaufmann, M., Weggen, S. \& Wirths, O. Limited effects of prolonged environmental enrichment on the pathology of 5XFAD mice. Mol. Neurobiol. 54, 6542-6555 (2017).

65. Wirths, O., Breyhan, H., Schäfer, S., Roth, C. \& Bayer, T. A. Deficits in working memory and motor performance in the APP/PS1ki mouse model for Alzheimer's disease. Neurobiol. Aging 29, 891-901 (2008).

66. Antunes, M. \& Biala, G. The novel object recognition memory: neurobiology, test procedure, and its modifications. Cogn. Process. 13, 93-110 (2012).

67. Jawhar, S., Trawicka, A., Jenneckens, C., Bayer, T. A. \& Wirths, O. Motor deficits, neuron loss, and reduced anxiety coinciding with axonal degeneration and intraneuronal $\mathrm{A} \beta$ aggregation in the 5XFAD mouse model of Alzheimer's disease. Neurobiol. Aging 33, 196.e29-40 (2012).

68. Sakib, M. S., Sokpor, G., Nguyen, H. P., Fischer, A. \& Tuoc, T. Intranuclear immunostaining-based FACS protocol from embryonic cortical tissue. STAR Protoc. 2, 100318 (2021).

69. Eisenberg, E. \& Levanon, E. Y. Human housekeeping genes, revisited. Trends Genet. 29, 569-574 (2013).

70. Becht, E. et al. Dimensionality reduction for visualizing single-cell data using UMAP. Nat. Biotechnol. 37, 38-44 (2018).

71. Ge, S. X., Jung, D. \& Yao, R. ShinyGO: a graphical gene-set enrichment tool for animals and plants. Bioinformatics 36, 2628-2629 (2020).

\section{Acknowledgements}

MK was supported by the Göttinger College for Translational Medicine by the Lower Saxony Ministry of Science and Culture. A.Ai. was supported by the European Research Council (ERC) Advanced Grant (ERCAdG No. 339580). M.S.S., A.F., P.R., A.Ai. and J.G. were funded by the Deutsche Forschungsgemeinschaft (DFG, German Research Foundation) under Germany's Excellence Strategy-EXC 2067/1-390729940, E.B. and G.H .by EXC2151-390873048 and S.N., C.St., and J.G. by TRR274. A.F. received funds from the ERC consolidator grant DEPICODE (648898), the BMBF project ENERGI

(01GQ1421A), the DFG SFB1286 and the Hans and Isle Breuer foundation. A.F. and J.G. had funds from the German Center for Neurodegenerative Diseases. S.N. (NE 2447/1-1) C.St. (STA1389/5-1) and J.G. (Ga354/16-1) were funded by DFG research grants. The authors thank the transgenic core facility of the Max Planck Institute of Experimental Medicine and especially Ursula Fünfschilling for microinjections and helpful discussions in generating the Rnaset $2^{-1-}$ mouse strain. We thank EUROIMMUN AG Lübeck, Germany for measuring of anti-nuclear antibodies (ANA), Hans-Joachim Anders for providing a positive control serum for ANA detection and Oliver Gross for urine protein analysis as well as Marc Ziegenbein, Elisabeth Ehbrecht, Susanne Burkhardt, Heidi Brodmerkel, and Olga Kowatsch for excellent technical assistance.

\section{Author contributions}

M.K., M.H., S.N. and J.G. designed and supervised the project. M.K., K.T., and K.W. performed and analyzed most experiments. S.Z. and O.W. performed the behavior testing in mice. C.Sch. performed the qPCR experiments for interferon-stimulated genes J.F., A.W., C.St. and S.N. designed and analyzed the neuropathology experiments. S.N. supported and performed part of the FACS analysis. A.Ai. and P.R. supported the CRISPR-Cas9 knockout mouse generation. K.W., S.H. and J.K. performed and analyzed histopathology, blood smear and bone marrow analysis. H.W. and S.S. supported the generation of RNT2A knockout mouse. S.B. and A.Al. performed and analyzed the MRI experiments. RE prepared tissue samples for single nucleus sequencing. M.S.S. isolated nuclei. M.S.S. and L.K. prepared sequencing libraries and performed next-generation sequencing. D.M.K. performed quality control, mapped and analyzed single nucleus sequencing data. D.M.K. and A.F. interpreted single nucleus sequencing data and A.F. generated the figures. M.K., K.T., S.N. and J.G. wrote the manuscript supported by K.W., M.P., E.B., G.H., M.H., O.W., A.F. and C.St. All authors read and approved the manuscript.

\section{Competing interests}

The authors declare no competing interests.

\section{Additional information}

Supplementary information The online version contains supplementary material available at https://doi.org/10.1038/s41467-021-26880-x.

Correspondence and requests for materials should be addressed to Matthias Kettwig.

Peer review information Nature Communications thanks the anonymous reviewer(s) for their contribution to the peer review of this work. Peer reviewer reports are available.

Reprints and permission information is available at http://www.nature.com/reprints

Publisher's note Springer Nature remains neutral with regard to jurisdictional claims in published maps and institutional affiliations.

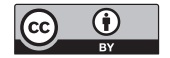

Open Access This article is licensed under a Creative Commons Attribution 4.0 International License, which permits use, sharing, adaptation, distribution and reproduction in any medium or format, as long as you give appropriate credit to the original author(s) and the source, provide a link to the Creative Commons license, and indicate if changes were made. The images or other third party material in this article are included in the article's Creative Commons license, unless indicated otherwise in a credit line to the material. If material is not included in the article's Creative Commons license and your intended use is not permitted by statutory regulation or exceeds the permitted use, you will need to obtain permission directly from the copyright holder. To view a copy of this license, visit http://creativecommons.org/ licenses/by/4.0/

(C) The Author(s) 2021 